Published in final edited form as:

Nat Metab. 2019 January ; 1(1): 47-57. doi:10.1038/s42255-018-0009-4.

\title{
Slc12a8 is a nicotinamide mononucleotide transporter
}

\author{
Alessia Grozio ${ }^{1}$, Kathryn F. Mills ${ }^{1}$, Jun Yoshino², Santina Bruzzone ${ }^{3}$, Giovanna Sociali ${ }^{3}$, \\ Kyohei Tokizane ${ }^{1}$, Hanyue Cecilia Lei ${ }^{1}$, Richard Cunningham ${ }^{4}$, Yo Sasaki ${ }^{5}$, Marie E. \\ Migaud $^{4}$, and Shin-ichiro Imai ${ }^{1},{ }^{*}$ \\ ${ }^{1}$ Department of Developmental Biology, Washington University School of Medicine,, St. Louis, MO \\ 63110, USA
}

${ }^{2}$ Center for Human Nutrition, Division of Geriatrics and Nutritional Science, Department of Medicine, Washington University School of Medicine, St. Louis, MO 63110, USA

${ }^{3}$ Department of Experimental Medicine, Section of Biochemistry, and Center of Excellence for Biomedical Research, University of Genova, 116132 Genova, Italy

${ }^{4}$ Mitchell Cancer Institute, University of South Alabama, Mobile, AL 36606, USA

${ }^{5}$ Department of Genetics, Washington University School of Medicine, St. Louis, MO 63110, USA

\section{Abstract}

\begin{abstract}
Nicotinamide mononucleotide (NMN) is a biosynthetic precursor of $\mathrm{NAD}^{+}$known to promote cellular $\mathrm{NAD}^{+}$production and counteract age-associated pathologies associated with a decline in tissue $\mathrm{NAD}^{+}$levels. How NMN is taken up into cells has not been entirely clear. Here we show that the $S 1 c 12 a 8$ gene encodes a specific NMN transporter. We find that Slc12a8 is highly expressed and regulated by $\mathrm{NAD}^{+}$in the murine small intestine. SIc12a8 knockdown abrogates the uptake of NMN in vitro and in vivo. We further show that Slc12a8 specifically transports NMN, but not nicotinamide riboside, and that NMN transport depends on the presence of sodium ion. Slc12a8 deficiency significantly decreases NAD ${ }^{+}$levels in the jejunum and ileum, which is associated with reduced NMN uptake as traced by doubly labeled isotopic NMN. Finally, we
\end{abstract}

Users may view, print, copy, and download text and data-mine the content in such documents, for the purposes of academic research, subject always to the full Conditions of use:http://www.nature.com/authors/editorial_policies/license.html\#terms

*Corresponding author Shin-ichiro Imai, M.D., Ph.D., Professor, Department of Developmental Biology, Department of Medicine (Joint), Washington University School of Medicine, Campus Box 8103, 660 South Euclid Avenue, St. Louis, MO 63110, USA, Tel: (314) 362-7228, Fax: (314) 362-7058, imaishin@wustl.edu.

Author contributions

S.I. conceived the project, and A.G. and S.I. mainly designed research, analyzed data, and wrote the manuscript. A.G. performed most experiments. K.F.M. performed the analyses of gut-specific Slc12a8 knockdown mice and whole-body Slc12a8KO mice with A.G.

J.Y. performed microarray analyses. S.B. and G.S. conducted proteoliposome experiments and analyzed the results with A.G. and S.I. K.T. and A.G. performed immunostaining, and H.C.L. and A.G. conducted Slc12a8 overexpression in vivo. R.C. and M.E.M. synthesized O18-D-NR and O18-D-NMN for this study, and Y.S. and A.G. conducted mass spectrometry analyses.

Author information

Reprints and permissions information is available at www.nature.com/reprints. Correspondence and requests for materials should be address to S.I. (imaishin@wustl.edu).

Data availability.The microarray data used in this study has been deposited into the NCBI GEO database (GEO accession numbers GSE49784 and GSE118365). All data generated or analyzed during this study are included in the article and its Supplementary Information.

Competing interests

A.G. and S.I. are inventors of a patent (PCT/US18/46233) about the Slc12a8 NMN transporter, whose applicant is Washington

University and which has been licensed by Teijin Limited (Japan). Other authors declare no competing financial interests. 
observe that 51 c12a8 expression is upregulated in the aged murine ileum, which contributes to the maintenance of ileal $\mathrm{NAD}^{+}$levels. Our work identifies the first NMN transporter and demonstrates that Slc12a8 has a critical role in regulating intestinal $\mathrm{NAD}^{+}$metabolism.

\section{Introduction}

It has been well documented that $\mathrm{NAD}^{+}$declines in many tissues during aging, including skeletal muscle, liver, adipose tissue, brain, pancreas, spleen, heart, kidney, and lung, contributing to the development of various age-associated pathophysiologies ${ }^{1-4}$. This phenomenon is caused, at least in part, by two molecular events: the age-associated decrease in $\mathrm{NAD}^{+}$biosynthesis mediated by nicotinamide phosphoribosyltransferase (NAMPT), the rate-limiting $\mathrm{NAD}^{+}$biosynthetic enzyme in mammals ${ }^{5}$, and the age-associated increase in $\mathrm{NAD}^{+}$consumption mediated by $\mathrm{NAD}^{+}$-consuming enzymes, such as poly-ADP-ribose polymerases ${ }^{6}$ and $\mathrm{CD} 38^{7}$. In mammalian $\mathrm{NAD}^{+}$biosynthesis, nicotinamide is a predominant precursor, and NAMPT catalyzes the conversion of nicotinamide and 5'phosphoribose pyrophosphate into nicotinamide mononucleotide (NMN), a key NAD ${ }^{+}$ intermediate ${ }^{8,9}$. NMN is also synthesized from nicotinamide riboside (NR), another NAD ${ }^{+}$ intermediate, by NR kinases, NRK1 and $2{ }^{10}$. NMN, together with ATP, is then converted into NAD ${ }^{+}$by NMN adenylyltransferases, NMNAT1-3. A number of studies have reported that NMN conveys remarkable effects of improving disease conditions and mitigating ageassociated physiological decline ${ }^{5,11-18}$. For example, NMN treatment is able to restore glucose-stimulated insulin secretion in aged C57BL/6 mice and some genetic mouse models that show reduced insulin-secreting capability 19,20 . NMN also enhances insulin sensitivity and secretion in mouse models of diet- and age-induced type 2 diabetes or obesity 5,11 . NMN has also been shown to prevent ischemia/reperfusion injury in the heart ${ }^{18}$. In addition, NMN maintains the neural stem/progenitor cell population in the aged hippocampus, improves mitochondrial function in aged skeletal muscle, and reverses arterial dysfunction in aged mice 12,13,16. In rodent models of Alzheimer's disease, NMN is able to protect mitochondrial and cognitive functions ${ }^{14,17}$. We have also demonstrated previously that NMN effectively mitigates age-associated physiological decline in regular chow-fed wildtype mice ${ }^{15}$. Collectively, these findings strongly suggest that NMN is a critical endogenous compound for $\mathrm{NAD}^{+}$biosynthesis and can be used as an efficient therapeutic and in preventive intervention against many age-associated disease conditions.

We have previously shown that NMN is absorbed from the gut into blood circulation within $2-3$ min and transported into tissues within $10-30 \mathrm{~min}^{5,15}$. NMN is then immediately utilized for $\mathrm{NAD}^{+}$biosynthesis, significantly increasing $\mathrm{NAD}^{+}$content in tissues over 60 $\mathrm{min}$. This fast pharmacokinetics has recently been confirmed by using doubly labeled isotopic NMN (C13-D-NMN), showing its rapid absorption and conversion to $\mathrm{NAD}^{+}$in peripheral tissues ${ }^{15}$. On the other hand, it has also been proposed that NMN is converted extracellularly to NR, which is transported into cells and reconverted to NMN ${ }^{21}$. Recent studies, however, have shown that the analyses of in vivo kinetics of these $\mathrm{NAD}^{+}$ intermediates are affected by differences in sample collection and extraction methodologies 22,23 (also see the Methods section). Therefore, it is critical to understand the mechanism by which NMN or NR is transported into cells or tissues. The fast pharmacokinetics of NMN 
led us to the hypothesis that there is an effective transporter that facilitates the direct uptake of NMN into the gut and other organs. Thus, we set out to identify this presumed NMN transporter in mammals.

\section{Results}

\section{Identification of a NMN transporter}

In our previous studies, we noticed that when NAMPT-mediated $\mathrm{NAD}^{+}$biosynthesis was inhibited by FK866, a potent NAMPT inhibitor, in various types of primary cells, coadministration of NMN always produced higher $\mathrm{NAD}^{+}$increases, compared to those that NMN induces in the absence of FK866 5,16,20. Thus, we hypothesized that the expression of a presumed NMN transporter might be upregulated when $\mathrm{NAD}^{+}$levels decrease. Based on this hypothesis, we conducted gene expression profiling in FK866-treated primary mouse hepatocytes, pancreatic islets, and hippocampal neurospheres, searching for genes commonly upregulated in these three primary cultures. We focused our searches to genes that encode transporters or transmembrane proteins and found only one gene that fitted these criteria but whose function was unknown. This gene, Slc12a8, exhibited 2.06, 1.69, and 4.91 for $\mathrm{Z}$ ratio in primary hepatocytes, islets, and neurospheres, respectively (Fig. 1a). The Slc12a8 gene belongs to the SLC12 gene family of the electroneutral cation-chloridecoupled cotransporters, and the function of the protein encoded by this gene remains unknown ${ }^{24}$. Close homologs exist in mouse, human, zebrafish, Drosophila, and C. elegans (Supplementary Fig. 1a). Although amino acid sequences are significantly diverged, the predicted ten membrane-spanning domains are conserved throughout these Slc12a8 homologs (Supplementary Fig. 1b). Slc12a 8 is highly expressed in the small intestine and pancreas and moderately in the liver and white adipose tissue (Fig. 1b). We confirmed that Slc12a8 expression was induced significantly in mouse primary hepatocytes, mouse NIH3T3 fibroblasts, and ex vivo explants of jejunum and ileum when $\mathrm{NAD}^{+}$was reduced by treatment with FK866, whereas this induction was suppressed when $\mathrm{NAD}^{+}$was restored by co-administration of FK866 and NMN (Fig. 1c and Supplementary Figs. 1c-d).

To begin to examine whether the S1c12a8 gene encodes the NMN transporter, we first determined the kinetics of NMN uptake in mouse primary hepatocytes. To inhibit the extracellular degradation of NMN to NR by CD73, the uptake of NR into cells through nucleoside transporters, and the intracellular NMN synthesis by NAMPT, we used AOPCP (adenosine- $5^{\prime}$-[a, $\beta$-methylene]diphosphate), dipyridamole, and FK866, respectively. AOPCP inhibits 5'-nucleotidase activity by $97 \%$ (Supplementary Fig. 1f). A cocktail of these inhibitors did not affect cell viability up to $30 \mathrm{~min}$ (Supplementary Fig. 1e). In the presence of these inhibitors, we added $100 \mu \mathrm{M}$ NMN and found that intracellular NMN levels significantly increased at 1 min time point compared to the control in mouse primary hepatocytes (Fig. 1d). In this condition using the same inhibitors and $100 \mu \mathrm{M}$ NMN, we knocked down Slc12a8 and Nrk1, a major NR kinase that converts NR to NMN intracellularly ${ }^{10}$, and examined the uptake of NMN at 1 min time point in primary hepatocytes. The knockdown (KD) efficiencies for both genes are approximately 80\% (Fig. 1e). Interestingly, the fast uptake of NMN was completely abrogated in Slc12a8-KD hepatocytes, whereas no significant reduction in NMN uptake was observed in Nrk1-KD 
hepatocytes (Fig. 1f), suggesting that Slc12a8 is necessary for the fast uptake of NMN in primary hepatocytes, and the observed increase in intracellular NMN is not due to the conversion of NR or nicotinamide into NMN.

\section{Biochemical features of the SIc12a8 protein}

Next, we overexpressed the full-length mouse SIc12a 8 cDNA in mouse NIH3T3 cells. We chose this cell line because it does not have any detectable extracellular activities of CD73 (converting NMN to NR) and CD38 (degrading NMN to nicotinamide and phosphoribose) and also has very weak NMN uptake activity (see Fig. 2b). The observed molecular weight of the full-length Slc12a8 protein is $\sim 90 \mathrm{kDa}$, which was also confirmed with N- and Cterminally FLAG-tagged Slc12a8 proteins (Supplementary Fig. 2a). Slc12a8 protein levels were significantly increased 2 2.2-fold in Slc12a8-overexpressing NIH3T3 (Slc12a8-OE) cells (Fig. 2a). We then determined the kinetics of NMN uptake using ${ }^{3} \mathrm{H}$-labeled NMN in Slc12a8-OE and control cells. The uptake of ${ }^{3} \mathrm{H}-\mathrm{NMN}$ was significantly enhanced at 3 and 5 min time points in Slc12a8-OE cells, compared to control cells (Fig. 2b). Using these Slc12a8-OE cells, we determined the Michaelis-Menten parameters for the Slc12a8 protein. The $K_{m}$ and the $V_{\max }$ for NMN were calculated to be $34.1 \pm 8.3 \mu \mathrm{M}$ and $11.5 \pm 1.2$ $\mathrm{pmol} / \mathrm{min} / \mathrm{mg}$, respectively (Fig. 2c). Notably, this $K_{m}$ is consistent with a detected range of NMN concentrations in mouse plasma and erythrocytes ${ }^{20,25,26}$. To further analyze the specificity of Slc12a8, we produced proteoliposomes by combining the membrane fractions of Slc12a8-OE or control NIH3T3 cells with the phospholipid bilayers derived from deproteinized erythrocyte plasma membrane. Slc12a8-OE-derived proteoliposomes incorporated significantly higher ( 2-fold) levels of ${ }^{3} \mathrm{H}-\mathrm{NMN}$ than those in control-derived proteoliposomes (Supplementary Fig. 2b). Using these Slc12a8-OE proteoliposomes, we examined whether the uptake of ${ }^{3} \mathrm{H}-\mathrm{NMN}$ into proteoliposomes would be competed by excess amounts of various cold $\mathrm{NAD}^{+}$-related compounds. $150 \mu \mathrm{M}$ of cold NMN showed complete competition against ${ }^{3} \mathrm{H}-\mathrm{NMN}$ uptake, whereas $\mathrm{NAD}^{+}, \mathrm{AMP}$, nicotinic acid mononucleotide (NaMN), and nicotinamide showed minimal or negligible competition at the same concentration (Fig. 2d). We confirmed that NaMN, a structurally very similar compound to NMN, was not transported into Slc12a8-OE proteoliposomes (Supplementary Fig. 2c). Interestingly, $150 \mu \mathrm{M}$ NR exhibited $\sim 70 \%$ displacement (Fig. 2d), and therefore, we further determined $\mathrm{IC}_{50}$ concentrations for $\mathrm{NMN}$ and $\mathrm{NR}$, using the proteoliposome system. The $\mathrm{IC}_{50}$ for $\mathrm{NMN}$ was $22.8 \pm 3.6 \mu \mathrm{M}$, whereas the $\mathrm{IC}_{50}$ for $\mathrm{NR}$ was $77.4 \pm 10.8$ $\mu \mathrm{M}$ (Fig. 2e). This result suggests that the Slc12a8 protein is specific primarily to NMN under physiological conditions because NR levels have not been shown to reach such high concentrations in pathophysiological conditions, such as in blood ${ }^{22,27}$ and ascitic exudates ${ }^{28}$. To definitively determine the specificity of Slc12a8 between NMN and NR, we gave doubly labeled, 3-Da heavier, isotopic NMN or NR (O18-D-NMN or O18-D-NR) to Slc12a8-OE and control NIH3T3 cells and measured these isotopic compounds transported into cells within $5 \mathrm{~min}$. When treating with $25 \mu \mathrm{M}$ O18-D-NMN, Slc12a8-OE cells showed approximately 4-fold higher uptake of O18-D-NMN compared to control cells but no increase in O18-D-NR, demonstrating unequivocal NMN uptake without any conversion of NMN to NR outside of cells within 5 min (Fig. 2f). On the other hand, when treating with 25 $\mu \mathrm{M}$ O18-D-NR, equivalent levels of O18-D-NMN synthesis and equivalent transport of O18-D-NR were detected in both Slc12a8-OE and control cells (Fig. 2f). These results 
clearly demonstrate that Slc12a8 specifically transports NMN, but not NR, in a minuteorder.

We next assessed the ion dependency of Slc12a8 for NMN transport, using the Slc12a8-OE proteoliposome system. When sodium was replaced with lithium, the ${ }^{3} \mathrm{H}-\mathrm{NMN}$ incorporation was dramatically reduced by $\sim 80 \%$ (Fig. $2 \mathrm{~g}$ ), indicating that NMN transport by Slc12a8 is sodium ion-dependent. Potassium ion is not sufficient to elicit the NMNtransporting function of Slc12a8 (Supplementary Fig. 2d), and chloride ion is not required for NMN transport (Fig. 2g), which distinguishes Slc12a8 from other known Slc12a family members that function as cation-chloride cotransporters ${ }^{24,29}$. Lastly, we compared the effect of Slc12a8 overexpression on $\mathrm{NAD}^{+}$biosynthesis in NIH3T3 cells. When control and Slc12a8-OE NIH3T3 cells were pre-treated for $1 \mathrm{hr}$ with a cocktail of $100 \mathrm{nM}$ FK866, $2 \mu \mathrm{M}$ dipyridamole, and $500 \mu \mathrm{M}$ AOPCP, intracellular $\mathrm{NAD}^{+}$levels were significantly reduced in both control and Slc12a8-OE cells (Fig. 2h). However, additional 1-hr incubation with 100 $\mu \mathrm{M}$ NMN was able to restore $\mathrm{NAD}^{+}$levels to the original levels only in Slc12a8-OE cells, not in control NIH3T3 cells (Fig. 2h). Furthermore, this Slc12a8-mediated restoration of $\mathrm{NAD}^{+}$was not affected by WNK463, a specific inhibitor for the WNK kinase that regulates the activity of the cation-chloride cotransporters ${ }^{29}$ (Supplementary Fig. 2e). All these results strongly support the function and the specificity of Slc12a8 as a novel NMN transporter in mammals.

\section{In vivo validation of the NMN transporter}

To further evaluate the NMN-transporting function of Slc12a8 in vivo, we first knocked down SIc12a8 in the small intestine, one of the tissues that have highest Slc12a8 expression, by giving oral gavages of lentiviruses carrying control firefly luciferase ( $f L u c$ ) shRNA or Slc12a 8 shRNA to young wild-type mice. Slc12a8 protein levels were reduced by $\sim 60 \%$ in the jejunum and $\sim 50 \%$ in the ileum in the mice receiving the Slc12a8 shRNA-expressing lentivirus in the gut, compared to the mice receiving the fLuc shRNA-expressing lentivirus (Fig. 3a). When orally administering NMN ( $500 \mathrm{mg} / \mathrm{kg}$ body weight) to those mice, plasma NMN levels significantly increased at $5 \mathrm{~min}$ in the control mice, whereas they did not increase at all in the Slc12a8-KD mice (Fig. 3b). Instead, plasma nicotinamide levels tended to be higher in Slc12a8-KD mice compared to control mice (Fig. 3c), probably because higher levels of NMN were subjected to degradation to nicotinamide in Slc12a8-KD mice. Consistent with these results, $\mathrm{NAD}^{+}$levels were significantly decreased in the jejunum of Slc12a8-KD mice compared to control mice (Fig. 3d). In the ileum, there were small $\mathrm{NAD}^{+}$ decreases, which did not reach statistical significance, in Slc12a8-KD mice (Fig. 3d). These results suggest that Slc12a8 in the small intestine is important to transport NMN from the gut into the circulation, affecting $\mathrm{NAD}^{+}$levels in the small intestine and the systemic NMN supply in vivo.

We also produced whole-body Slc12a8 knockout (Slc12a8KO) mice by excising the exon 4 of the SIc12a8 gene using the CRISPR-CAS9 system. The birth ratio of these knockout mice was lower than the expected Mendelian ratio, implying that there was some premature death during the embryonic stage. However, the pups that were born safely were able to grow to adults, and they did not show any gross abnormalities. In these adult Slc12a8KO mice, we 
confirmed that the expression of the full-length Slc12a8 mRNA was completely abolished in tissues (Supplementary Fig. 3a). We also confirmed that the Slc12a8 protein was abolished in the jejunum, ileum, and pancreas of the Slc12a8KO mice by Western blotting (Fig. 3e and Supplementary Fig. 3b). The duodenum does not express the Slc12a8 protein (Fig. 3e), even though it expresses high levels of Slc12a8 mRNA (Fig. 1b). Immunostaining signals of Slc12a8 in the gut, which are detected predominantly in apical, lateral, and basal membranes of the villi in the jejunum and ileum of wild-type mice, were abolished in Slc12a8KO mice (Fig. 3f and Supplementary Fig. 3c), confirming the localization of Slc12a8 in the gut epithelia. Consistent with these findings, the S/c12a8KO mice showed significant decreases in $\mathrm{NAD}^{+}$levels in the jejunum and ileum, but not in the duodenum, particularly during the dark time when NAD ${ }^{+}$levels usually rise (Fig. 3g and Supplementary Fig. 3d). NAD ${ }^{+}$ decreases were also detected in the pancreas during both light and dark times (Supplementary Fig. 3d). To further confirm whether NMN transport is compromised in the small intestine of the S1c12a8KO mice, we conducted a gavage of doubly labeled, isotopic NMN (O18-D-NMN) and measured the direct uptake of O18-D-NMN into the jejunum and ileum. At 10 min after administering O18-D-NMN by oral gavage, we clearly detected O18D-NMN in the wild-type jejunum and ileum, whereas the uptake of O18-D-NMN decreased by $46 \%$ and $36 \%$ in the jejunum and ileum of the Slc12a8KO mice, respectively (Fig. 3h).

Lastly, by using primary hepatocytes from control and Slc12a8KO mice, we again compared the transport of O18-D-NMN and O18-D-NR. When treating with $100 \mu \mathrm{M}$ O18-D-NMN, Slc12a8KO hepatocytes showed $\sim 90 \%$ reduction in O18-D-NMN uptake, compared to control wild-type hepatocytes at $5 \mathrm{~min}$ (Fig. 3i). Both cells showed no detectable levels of O18-D-NR (Fig. 3i). On the other hand, when treating with $100 \mu \mathrm{M}$ O18-D-NR, equivalent levels of O18-D-NMN synthesis were detected in both Slc12a8KO and control hepatocytes, although there appeared to be some compensatory increases in O18-D-NR uptake in Slc12a8KO hepatocytes (Fig. 3i). These results provide strong support for the specificity and the minute-order kinetics of Slc12a8 as a novel NMN transporter.

\section{SIc12a8 maintains $\mathrm{NAD}^{+}$levels in the aged gut}

It has been well documented that $\mathrm{NAD}^{+}$decreases over age in multiple tissues ${ }^{4}$. We found that the jejunum and ileum in 24 month-old mice also showed $\mathrm{NAD}^{+}$decreases, compared to 2 month-old mice, although the difference did not reach statistical significance in the jejunum (Fig. 4a). Consistent with this phenomenon, Slc12a8 expression was also significantly upregulated in the aged ileum (Fig. 4b). To investigate the biological relevance of this S1c12a8 upregulation in the aged ileum, we first gave an oral gavage of NMN to young ( 3 month-old) and aged ( 26 month-old) wild-type mice. Although the absolute plasma NMN levels were lower throughout all time points in aged mice compared to young mice (Fig. 4c), the fold increases of plasma NMN levels were higher in aged mice compared to young mice (Fig. 4d). NAD ${ }^{+}$levels were also increased in aged mice to the levels close to young mice after NMN oral gavage (Fig. 4e), and their fold increases were equivalent between young and aged mice (Fig. 4f), indicating that the upregulation of Slc12a8 plays an important role in counteracting age-associated $\mathrm{NAD}^{+}$decline in the small intestine. To further test this possibility, we conducted oral gavages of lentiviruses carrying control $f L u c$ shRNA and Slc12a8 shRNA to 2- and 24-month-old mice and examined their NAD ${ }^{+}$levels 
in the gut. Knockdown efficiencies were similar between young and aged mice (Supplementary Figs. 4a-b). Significant $\mathrm{NAD}^{+}$decreases were detected in the ilea of the aged, but not young, Slc12a8-KD mice (Fig. 4g). Luminal NMN content in the jejunum and ileum did not show any significant differences between young and aged mice (Supplementary Fig. 4c). Contrarily, we also overexpressed SIc12a8 in the small intestine of young wild-type mice by giving oral gavages of the Slc12a8-expressing lentivirus. The Slc12a8 protein was overexpressed $\sim 1.5$-fold in the ilea (Supplementary Fig. 4 d), which induced significant $\mathrm{NAD}^{+}$increases in the ileum (Supplementary Fig. 4e). Taken together, these results demonstrate that the upregulation of S/c12a8 in the ileum contributes to the maintenance of $\mathrm{NAD}^{+}$levels in the aged intestine.

\section{Discussion}

We have previously demonstrated that the transport of NMN from the gut to the circulation and then to tissues occurred within $10 \mathrm{~min}^{15}$. However, the mechanism that mediates such minute-order transport of NMN has so far remained unknown. In this study, we demonstrate that the Slc12a8 gene encodes a novel NMN transporter in mammals. The mRNA expression of the Slc12a8 gene is upregulated in response to $\mathrm{NAD}^{+}$decline, allowing cells to meet to an urgent demand of $\mathrm{NAD}^{+}$biosynthesis. The Slc12a8 NMN transporter is specific to NMN and requires the sodium ion for the transport of NMN. The abrogation or the deficiency of Slc12a8 in cell culture and in the small intestine significantly reduces the uptake of NMN, resulting in reduced $\mathrm{NAD}^{+}$levels in the jejunum and ileum in vivo. Conversely, cellular and intestinal overexpression of full-length $S / c 12 a 8$ provides a full capacity of NMN transport to the cells that otherwise exhibit minimal NMN transport and also increases ileal NAD ${ }^{+}$levels in vivo, respectively. Furthermore, the whole-body SIc12a8KO mice display significant defects in direct, minute-order NMN transport and NAD ${ }^{+}$biosynthesis, particularly in the jejunum and ileum. In the aged ileum, Slc12a8 expression is upregulated in response to decreased $\mathrm{NAD}^{+}$content. Perturbation of this Slc12a8 upregulation in the aged ileum affects homeostatic regulation of ileal $\mathrm{NAD}^{+}$, causing further decreased NAD ${ }^{+}$in the aged ileum. Thus, this newly identified NMN transporter encoded by the SIc12a8 gene, namely, the transmembrane nucleotide transporter identified for the first time in mammals, functions to regulate $\mathrm{NMN}$-driven $\mathrm{NAD}^{+}$biosynthesis and maintain intestinal $\mathrm{NAD}^{+}$levels in aged individuals.

How NMN is transported into cells has long been a matter of debate in the field of NAD ${ }^{+}$ biology. It has been believed that NMN needs to be converted first to NR by CD73 outside of cells and then NR is transported into cells, likely through the nucleoside transporter, and reconverted to NMN by NRK1/2 ${ }^{21,30}$. Although this process can occur over the course of $24 \mathrm{~h}^{21}$, such hourly kinetics cannot explain the minute-order uptake of NMN into the cell. Additionally, how to analyze the in vivo kinetics of NMN is also critical, and the results could be significantly affected by differences in sample collection and extraction methodologies 22,23 . For example, plasma samples need to be processed immediately after collection, as we did in this study, because freezing blood or plasma samples causes inaccurate measures of NMN levels. The results presented in this study strongly indicate that Slc12a8 is specific to NMN, not to NR, and its $K_{m}$ is consistent with a measured range of NMN concentrations ${ }^{20,25,26}$. Even NaMN, structurally very close to NMN, cannot be 
transported by Slc12a8. Furthermore, its dependency on sodium ion, but not chloride or potassium ions, and its insensitivity to WNK463 distinguish Slc12a8 from other known cation-chloride cotransporters. Nonetheless, because a very high, supra-physiological concentration of NR can compete against NMN, NR might be able to interact with Slc12a8 weakly. To elucidate the precise details of the structure-function relationship between Slc12a8 and NMN, it will be of great importance to determine the crystal structure of the Slc12a8 NMN transporter.

The results from gut-specific Slc12a8-KD mice and whole-body SIc12a8KO mice demonstrate that the major place where NMN is absorbed is the small intestine, particularly the jejunum and ileum. Whereas we can detect NMN in the luminal content of the jejunum and ileum, the source of NMN remains unknown at this moment. For humans, certain vegetables and fruits contain NMN ${ }^{15}$. Additionally, it has been reported that human and cow milk contains NMN at micromolar concentrations ${ }^{31,32}$. For mice, one interesting possibility is that NMN could be produced from the enzymatic degradation of $\mathrm{NAD}^{+}$in the small intestine. Another possibility is that certain gut bacterial species may produce NMN. If this is the case, the Slc12a8 NMN transporter would likely play a critical role in the symbiotic regulation of $\mathrm{NAD}^{+}$biosynthesis between the microbiota and the host.

Remarkably, the function of the Slc12a8 NMN transporter becomes crucial in aged individuals compared to young ones. In response to significant decreases in $\mathrm{NAD}^{+}$levels, the aged ileum upregulates $S / c 12 a 8$ expression and tries to maintain its $\mathrm{NAD}^{+}$levels. When enough NMN is supplied, this feedback system can function adequately to maintain comparable levels of $\mathrm{NAD}^{+}$to those in young ilea. Therefore, increasing NMN availability or stimulating the function of the NMN transporter could effectively counteract ageassociated $\mathrm{NAD}^{+}$decline in the aged small intestine. In addition to aging, the NMNtransporting function of Slc12a8 might also be important for the pathogenesis of some diseases. For example, the human SLC12A8 gene has been identified as a psoriasis susceptibility candidate gene ${ }^{33}$. Most recently, it has been reported that the high expression of SLC12A8 and its genetic polymorphism are associated with better prognosis of patients with pancreatic ductal adenocarcinomas ${ }^{34}$ and breast cancers ${ }^{35}$, respectively. Thus, the Slc12a8 NMN transporter could be a new, interesting target for pharmaceutical drug development.

In conclusion, the identification and the characterization of the Slc12a8 NMN transporter further advances our understanding of the physiological importance of NMN as a key systemic $\mathrm{NAD}^{+}$intermediate. Because NMN conveys remarkable effects of mitigating ageassociated physiological decline in mice ${ }^{4,15}$, identifying compounds that could promote the NMN-transporting function of the Slc12a8 protein will provide an interesting opportunity to develop a more effective anti-aging intervention, combined with NMN administration.

\section{Methods}

\section{Microarray Analysis.}

Total RNA was isolated from primary hepatocytes, pancreatic islets, and hippocampal neurospheres, treated with $0.1 \%$ DMSO (control) or FK866 (200 nM for primary 
hepatocytes, and $10 \mathrm{nM}$ for pancreatic islets and hippocampal neurospheres). Primary hepatocytes and pancreatic islets were isolated from 3 month-old C57BL/6J (B6) male mice (Jackson Laboratories). To determine transcriptional changes induced by FK866 treatment, microarray analyses were conducted using the Illumina Mouse Ref 8 whole genome microarrays (version 2). The background-subtracted raw microarray data were subjected to $\mathrm{Z}$ score transformation, and $\mathrm{Z}$ ratios were calculated as described previously ${ }^{5}$. All data were analyzed by the R statistical software package.

\section{Cell culture and ex vivo small intestine explant culture.}

NIH3T3 cells (originally purchased from the American Type Culture Collection [ATCC]) were cultured at $37{ }^{\circ} \mathrm{C}$ and $5 \% \mathrm{CO}_{2}$ in Dulbecco's modified Eagle's medium (DMEM) supplemented with $10 \%$ fetal bovine serum (FBS), 100 units $/ \mathrm{ml}$ penicillin, and $100 \mu \mathrm{g} / \mathrm{ml}$ streptomycin. For Slc12a8 mRNA expression analysis, $2.5 \times 10^{5}$ cells per well were incubated in 6-well plates with DMEM with $1 \%$ FBS containing $0.1 \%$ DMSO or $100 \mathrm{nM}$ FK866 or $100 \mathrm{nM}$ FK866 plus $100 \mu \mathrm{M}$ NMN for 24h. Small intestines from 3 month-old C57BL/6J (B6) male mice (Jackson Laboratories) were cut into three segments with duodenum/jejunum/ileum length ratios of 1:3:2 ${ }^{36}$. One centimeter of each segment was opened longitudinally, washed once with cold PBS, and incubated for $4 \mathrm{~h}$ at $37^{\circ} \mathrm{C}$ with $0.1 \%$ DMSO or $100 \mathrm{nM}$ FK866 or $100 \mathrm{nM}$ FK866 plus $500 \mu \mathrm{M}$ NMN in the 1:1 mixture of DMEM and Ham's F-12 medium (Sigma) with 5\% FBS and the following additives: 5 $\mu \mathrm{g} / \mathrm{ml}$ insulin (Sigma), $20 \mathrm{ng} / \mathrm{ml}$ epidermal growth factor (Sigma), 1x B27 supplement (GIBCO), $1 \mathrm{mM}$ Sodium pyruvate (Corning), $100 \mathrm{units} / \mathrm{ml}$ penicillin, $100 \mu \mathrm{g} / \mathrm{ml}$ streptomycin, and $2 \mathrm{mM}$ glutamax (GIBCO). Cellular and tissue total RNA samples, which were extracted using the PureLink RNA Mini kit (Cat\# 12183025, Ambion), were analyzed by quantitative RT-PCR, and relative expression levels were calculated for each gene by normalizing to Gapdh expression levels ${ }^{16}$.

\section{NAD+ and NMN measurements by HPLC.}

$\mathrm{NAD}^{+}$and $\mathrm{NMN}$ were extracted from cells and tissues with perchloric acid, neutralized with $\mathrm{K}_{2} \mathrm{CO}_{3}$ on ice, and quantitated by our high-performance liquid chromatography (HPLC) system (Shimadzu) with a Supelco LC-18-T column $(15 \mathrm{~cm} \times 4.6 \mathrm{~cm}$; Sigma) and a Hypercarb column $(15 \mathrm{~cm} \times 4.6 \mathrm{~cm}$; Thermo Scientific $)$, respectively 5,37 .

\section{Flow cytometry analysis.}

$2 \times 10^{6}$ NIH3T3 cells were incubated in a 10-cm culture dish with DMEM with $1 \%$ FBS containing $0.1 \%$ DMSO or $100 \mathrm{nM}$ FK866 or $100 \mathrm{nM}$ FK866 plus $100 \mu \mathrm{M}$ NMN for $48 \mathrm{~h}$ at $37^{\circ} \mathrm{C}$ and $5 \% \mathrm{CO}_{2}$. Cells were then washed once with cold PBS, treated with $0.02 \%$ EDTA in PBS, and stained for flow cytometry using a commercially available polyclonal rabbit anti-mouse Slc12a8 antibody (ARP44039, Aviva, CA) at 1:200, a secondary goat anti-rabbit $\operatorname{IgG}(\mathrm{H}+\mathrm{L})$ conjugated with Alexa Fluor 488 at 1:2000 (Invitrogen), and the survival marker Zombie Dye at 1:400 (Biolegend) for $25 \mathrm{~min}$ at $4^{\circ} \mathrm{C}$. Cells were then washed and analyzed by the Gallios Flow Cytometer (BeckmanCoulter). For the intracellular staining, cells were first fixed in 2\% PFA for $10 \mathrm{~min}$ at room temperature and then permeabilized in saponincontaining buffer for another $10 \mathrm{~min}$ at RT. Slc12a8 staining was performed in permeabilization buffer for $25 \mathrm{~min}$ at $4^{\circ} \mathrm{C}$. Samples were analyzed by the Gallios Flow 
Cytometer, and data were analyzed using Kaluza 1.3 (BeckmanCoulter). Dead cells were excluded using a Zombie Aqua Fixable Viability Kit (Biolegend).

\section{Hepatocytes isolation, 5'-nucleotidase activity assay, NMN uptake measurement, and silencing of S/c12a8 and Nrk1 expression.}

Primary hepatocytes were isolated from 3 month-old B6 male mice (Jackson Laboratories) by a two-step hepatic portal perfusion with calcium and magnesium-free Hanks' salt solution followed by DMEM containing $0.25 \mathrm{mg} / \mathrm{mL}$ collagenase (Type IV, Cat \# C5138, Sigma) ${ }^{38}$. Cells were cultured overnight in 6-well plates coated with poly-L-lysine at $37^{\circ} \mathrm{C}$ and 5\% $\mathrm{CO}_{2}$ in DMEM supplemented with $10 \% \mathrm{FBS}, 100 \mathrm{units} / \mathrm{ml}$ penicillin, and 100 $\mu \mathrm{g} / \mathrm{ml}$ streptomycin (pen/strep) before conducting any experiments. Slc12a8 mRNA expression and $\mathrm{NAD}^{+}$content were evaluated by incubating hepatocytes with $500 \mathrm{nM}$ FK866 or $500 \mathrm{nM}$ FK866 plus $500 \mu \mathrm{M}$ NMN in DMEM with 1\% FBS for 24h. To make sure whether adenosine- $5^{\prime}$-[a, $\beta$-methylene]diphosphate (AOPCP) inhibits 5 '-nucleotidase activity, $1.5 \times 10^{5}$ cells per well were grown in 12-well plates with $500 \mathrm{nM}$ FK866 in DMEM with $1 \%$ FBS for 24h and then incubated in $1.4 \mathrm{ml}$ of Hanks' buffered saline solution with $\mathrm{Ca}^{2+}$ and $\mathrm{Mg}^{2+}$ at $\mathrm{pH} 7.5$ (HBSS, GIBCO) in the presence of $100 \mu \mathrm{M}$ adenosine monophosphate (AMP) or $100 \mu \mathrm{M}$ AMP plus $500 \mu \mathrm{M}$ AOPCP. At different time points $(0,1,5,15$, and $30 \mathrm{~min}), 200 \mu \mathrm{l}$ of each culture supernatant were collected and extracted by adding $28 \mu \mathrm{l}$ of $70 \%$ perchloric acid. The amounts of adenosine produced were determined by HPLC. Elution times for AMP and adenosine were 4.7 and $17.4 \mathrm{~min}$, respectively. To examine cell viability, Celltiter 96 AQueous One Solution Cell Proliferation Solution (Promega, MA) was used, and the absorbance was measured at $\lambda=490$ after $4 \mathrm{~h}$ incubation. For NMN uptake measurement, $1.5 \times 10^{5}$ cells per well were grown in 12-well plates with $500 \mathrm{nM}$ FK866 in DMEM with 1\% FBS for 24h and then incubated in $1 \mathrm{ml}$ of HBSS in the presence of $500 \mu \mathrm{M}$ AOPCP, $20 \mu \mathrm{M}$ dipyridamole, and $500 \mathrm{nM}$ FK866 or these inhibitors plus $100 \mu \mathrm{M} N \mathrm{NMN}$. At different time points $(0,0.25,1,5,15$, and $30 \mathrm{~min})$, cells were washed once with cold HBSS and lysed in cold $10 \%$ perchloric acid. Intracellular NMN levels were measured by HPLC as described previously ${ }^{5}$. For gene silencing experiments, $10 \mu \mathrm{g}$ of ON-TARGET plus mouse siRNA (Thermo scientific) specific to Slc12a8 (J-042450-12-0020) or Nrk1 (J 051839-11-0010) or a negative control siRNA (NON-targeting siRNA \#1, D-001810-01-20) were electroporated into one million cells per condition, mixed with $100 \mu \mathrm{l}$ AMAXA Mouse Hepatocyte Nucleofector Solution (Lonza), using the Nucleofector program H-26 following the manufacturer's instructions. The electroporated cells were incubated in the cuvette for $15 \mathrm{~min}$ before addition of media. $2.5 \times 10^{5}$ cells per well were seeded in 6 -well plates coated with poly-L-lysine at $37^{\circ} \mathrm{C}$ and $5 \% \mathrm{CO}_{2}$ in DMEM containing 10\% FBS and penicillin-streptomycin for 48h after electroporation. Those cells were incubated with $500 \mathrm{nM}$ FK866 in DMEM with 1\% FBS for $24 \mathrm{~h}$. NMN uptake was measured by HPLC after incubating cells in HBSS with $500 \mu \mathrm{M}$ AOPCP, $20 \mu \mathrm{M}$ dipyridamole, and $500 \mathrm{nM}$ FK866 or these inhibitors plus $100 \mu \mathrm{M}$ NMN for $1 \mathrm{~min}$ at room temperature. Silencing efficiencies were evaluated by quantitative RT-PCR.

\section{Generation of NIH3T3 cells stably overexpressing the full-length mouse SIc12a8 cDNA.}

The coding region of full-length mouse SIc12a 8 cDNA (GenBank Reference Sequence: NM_134251) was amplified from mouse liver by PCR using PfuUltra II Fusion HS DNA 
polymerase (Agilent, CA) with the following forward and reverse primers containing XhoI sites:Slc12a8 forward: 5'-ATACTCGAGGAGAATGGCCCAGAGGTCTC-3', Slc12a8 reverse: 5'- TCAACTACGGAGGGATGATCGAGCTCATT -3'

The resulting 2118-bp fragment of full-length SIc12a8 cDNA was digested with XhoI and cloned into pBluescript SK- vector. SIc12a8 cDNA fragment was then subcloned into the mammalian expression vector pCXN2 ${ }^{39}$. N- or C-terminally FLAG-tagged versions of fulllength Slc12a8 cDNA were produced using the following forward and reverse primer sets containing XhoI sites and FLAG-tag sequences:, N-terminally FLAG-tagged Slc12a8, Forward: 5'-

ATACTCGAGCCACCATGGACTACAAAGACGATGACGACAAGGGCGCCCAGAGGTC TCCG-3'Reverse: 5' - TCAACTACGGAGGGATGATCGAGCTCATT -3'C-terminally FLAG-tagged Slc12a8Forward: 5'- ATACTCGAGCCACCATGGCCCAGAGGTCTCCG -3'Reverse: 5'-

TCAACTACGGAGGGATGCCGCTGATGTTTCTGCTACTGCTGTTCATCGAGCTCATT $-3^{\prime}$

The resultant FLAG-tagged Slc12a8 cDNA fragments were cloned into the mammalian expression vector pIRES-EGFP-puro (Addgene, Cat\#45567). The Slc12a8cDNA sequence in the final vector was confirmed by sequencing. NIH3T3 cells were transfected with $5 \mu \mathrm{g}$ of pCXN2 carrying the full-length Slc12a8 cDNA (Slc12a8-OE) or only pCXN2 vector (control) or $5 \mu \mathrm{g}$ of pIRES-EGFP-puro carrying the full-length Slc12a8 cDNA with N-or Cterminal FLAG-tag (Slc12a8-OE) or only pIRES-EGFP-puro vector (control) using the SuperFect transfection reagent (QIAGEN) and cultured in DMEM supplemented with 10\% FBS, antibiotics, and $300 \mu \mathrm{g} / \mathrm{ml} \mathrm{G} 418$ (Invitrogen) or $1 \mu \mathrm{g} / \mathrm{ml}$ puromycin (Sigma, MO) for 2 weeks. Resistant cells were pooled, and aliquots were frozen for further experiments. To confirm Slc12a8 protein expression levels, plasma membrane (PM) fractions were prepared from control and Slc12a8-OE cells or N- or C-terminally FLAG-tagged Slc12a8-OE cells, as described previously ${ }^{40}$. Briefly, $7.5 \times 10^{7}$ cells cultured in five $10-\mathrm{cm}$ dishes were used. After 2 washes with ice-cold HES buffer (20 mM HEPES, $1 \mathrm{mM}$ EDTA, and $255 \mathrm{mM}$ sucrose, $\mathrm{pH}$ 7.4), cells were collected by scraping in HES buffer ( $3 \mathrm{ml} / \mathrm{dish}$ ) containing a protease inhibitor cocktail (Roche) and homogenized by passing 5 times through a 22-gauge needle. All subsequent steps were performed at $4^{\circ} \mathrm{C}$. The homogenate was centrifuged (Avanti J-E; Beckman-Coulter) at 10,000 $\mathrm{g}$ in a JA 25.5 rotor for $15 \mathrm{~min}$. The resulting supernatant was layered on the top of a 10-ml sucrose cushion (38.5\% sucrose, $20 \mathrm{mM}$ HEPES, and $1 \mathrm{mM}$ EDTA, pH 7) and centrifuged at 53,000 g for $120 \mathrm{~min}$. The interface containing the PM fraction was carefully removed, resuspended in $10 \mathrm{ml}$ HES buffer, and centrifuged at 50,000 $\mathrm{g}$ for $30 \mathrm{~min}$, yielding the PM fraction in the pellet. PM fractions from control or Slc12a8OE cells were lysed with RIPA buffer (150 mM sodium chloride, $1.0 \%$ NP-40, $0.25 \%$ sodium deoxycholate, $0.1 \%$ SDS, $50 \mathrm{mM}$ Tris, $\mathrm{pH}$ 7.5, 2 mM EDTA, $1 \mathrm{mM}$ PMSF, 0.5mM DTT and protease inhibitor cocktail) and boiled for 5 minutes in 1x Laemmli buffer. Western blotting was conducted with rabbit polyclonal anti-mouse Slc12a8 (1:500, ARP44039, Aviva, CA) or monoclonal anti-FLAG MS (1:500, \#F3165, Sigma, MO), and antiCaveolin-1 (1:2000, \#3238, Cell Signaling, MA) antibodies. Band intensity was quantitated on the Amersham Hyperfilm ${ }^{\mathrm{TM}}$ ECL (GE Healthcare limited) by Photoshop. To measure intracellular $\mathrm{NAD}^{+}$levels, $2 \times 10^{5}$ control and Slc12a8-OE NIH3T3 cells were preincubating 
with $2 \mu \mathrm{M}$ dipyridamole, $500 \mu \mathrm{M}$ AOPCP, $100 \mathrm{nM}$ FK866, and in some experiments, $10 \mu \mathrm{M}$ WNK463, a pan-WNK kinase inhibitor (MCE, \# HY-100626), for $1 \mathrm{~h}$ and then with the same inhibitors combined with $100 \mu \mathrm{M}$ NMN for an additional $1 \mathrm{~h}$ in DMEM $1 \% \mathrm{FBS}$ at $37^{\circ} \mathrm{C}$. At the end of incubation, cells were washed once with cold PBS and lysed in $10 \%$ perchloric acid. $\mathrm{NAD}^{+}$levels were determined by HPLC, as described above.

\section{NMN uptake analyses with radiolabeled NMN.}

Control and Slc12a8-OE NIH3T3 cells were harvested by centrifugation (400g, $5 \mathrm{~min})$, washed once in HBSS, and incubated at $37^{\circ} \mathrm{C}$ in HBSS [pH 7.5] $\left(5 \times 10^{6}\right.$ cells $\left./ \mathrm{ml}\right)$ containing $100 \mathrm{nM}^{3} \mathrm{H}-\beta$-nicotinamide mononucleotide ( $9 \mathrm{Ci} / \mathrm{mmol}$; Moravek Biochemicals, $\mathrm{CA}$ ) and unlabeled NMN to make the final concentration $25 \mu \mathrm{M}$. At the designated time points $(1,3$, 5 and 10 minutes), aliquots of the cells $(100 \mu \mathrm{l})$ were collected and placed in $1.5-\mathrm{ml}$ microcentrifuge tubes containing silicone-mineral oil (density, 1.015; Sigma-Aldrich) on the top of $2 \mathrm{M}$ potassium hydroxide solution, followed by centrifugation at $16,000 \mathrm{~g}$ for $30 \mathrm{~s}$. Cells were separated from the buffer and pelleted through the silicone-mineral oil layer. The radioactivity in these cell pellets was determined by a liquid scintillation counter. For calculating $K_{m}$ and $V_{\max }$, we used the same condition described above, but with various total concentrations of NMN, ranging from $1 \mu \mathrm{M}$ to $100 \mu \mathrm{M}$. At the 4-min time point, $100 \mu \mathrm{l}$ of cell suspension was collected and pelleted in the same way described above. The radioactivity in cell pellets was determined by a liquid scintillation counter. The radioactivity measured in control NIH3T3 cells was subtracted to calculate Slc12a8-specific NMN uptake in Slc12a8-OE NIH3T3 cells.

\section{Proteoliposome experiments.}

Proteoliposome preparation was carried out as previously described ${ }^{41}$. Briefly, $2 \times 10^{7}$ control or Slc12a8-OE NIH3T3 cells were resuspended in $1 \mathrm{ml}$ of lysis buffer $(10 \mathrm{mM}$ Tris$\mathrm{HCl}, \mathrm{pH} 8.3,150 \mathrm{mM} \mathrm{NaCl}, 0.3 \mathrm{M}$ sucrose, protease inhibitor cocktail) and disrupted using a homogenizer. The lysate was centrifuged for $10 \mathrm{~min}$ at $3,000 \mathrm{~g}$, and the supernatant was collected and centrifuged for $15 \mathrm{~min}$ at 100,000g. The membrane proteins were solubilized with buffer A (10 mM Tris- $\mathrm{HCl}$ [pH 8.3] containing $150 \mathrm{mM} \mathrm{NaCl}$ and $0.5 \%$ n-octyl- $\beta$ glucopyranoside). Separately, total lipids were extracted from hemoglobin-free erythrocyte membranes (ghosts) as described previously ${ }^{42}$. Total lipids from human erythrocyte membranes $(3 \mathrm{mg}$ ) were dried and resuspended with $600 \mu \mathrm{l}$ of solubilized membrane proteins (at approximately $0.7 \mathrm{mg} / \mathrm{ml}$ protein concentration). The resulting emulsions were sonicated in ice for $1 \mathrm{~min}$ and dialyzed against 5 liters of buffer A without n-octyl- $\beta$ glucopyranoside (dialysis buffer) for $24 \mathrm{~h}$ at $4^{\circ} \mathrm{C}$. Proteoliposomes were recovered, centrifuged for $15 \mathrm{~min}$ at $100,000 \mathrm{~g}$, resuspended in $900 \mu \mathrm{l}$ dialysis buffer and passed 5 times through a 30-gauge needle. To examine the $\mathrm{Na}^{+}-\mathrm{K}^{+}$- or $\mathrm{Cl}^{-}$-dependency of Slc12a8, $\mathrm{NaCl}$ was replaced with $150 \mathrm{mM} \mathrm{LiCl}, \mathrm{KCl}$, or $\mathrm{CH}_{3} \mathrm{COONa}$, respectively. All steps were carried out at $4^{\circ} \mathrm{C}$. Proteoliposomes ( $30 \mu \mathrm{l}$ in triplicate for each condition) were incubated for 2,5 , and $10 \mathrm{~min}$ at $25^{\circ} \mathrm{C}$ in the presence of $150 \mathrm{nM}\left(10^{5} \mathrm{cpm} / \mathrm{ml}\right){ }^{3} \mathrm{H}-\beta-\mathrm{NMN}$ (specific activity, $9.1 \mathrm{Ci} / \mathrm{mmole}$ ) with or without $150 \mu \mathrm{M}$ label-free compounds (NMN, NR, NAD, AMP, nicotinic acid mononucleotide [NaMN], and nicotinamide). At the end of incubations, samples were filtered on a glass fiber paper. Filters were washed with $3 \mathrm{ml}$ of dialysis buffer, dried, and counted for radioactivity. In each experiment, we always measured the non- 
specific binding/uptake of ${ }^{3} \mathrm{H}-\mathrm{NMN}$ by adding $150 \mathrm{nM}{ }^{3} \mathrm{H}-\mathrm{NMN}$ and $300 \mu \mathrm{M}$ of cold NMN (2000-fold higher concentration) to the proteoliposomes derived from Slc12a8-OE NIH3T3 cells and measuring the radioactivity of ${ }^{3} \mathrm{H}-\mathrm{NMN}$ after extensive washes. Because specific NMN transport should be displaced completely by the 2000-fold higher concentration of cold NMN, ${ }^{3} \mathrm{H}-\mathrm{NMN}$ detected in this condition must be due to a non-specific/non-kinetic transport and/or a non-specific binding to proteoliposomes. Such values due to non-specific transport/biding of ${ }^{3} \mathrm{H}-\mathrm{NMN}$ were always measured in each experiment and subtracted from all measured values of ${ }^{3} \mathrm{H}-\mathrm{NMN}$ transported into proteoliposomes in each condition. To examine whether Slc12a8 could transport NaMN, proteoliposomes (300 $\mu$ in triplicate for each condition) were incubated for 5 min with $5 \mathrm{mM}$ NMN or NaMN (Sigma, \#N7764). Right after incubation, samples were filtered on a glass fiber paper $\left(0.25 \mathrm{~cm}^{2}\right.$ area $)$, and nucleotides were extracted with $200 \mu \mathrm{l}$ of $0.6 \mathrm{M}$ perchloric acid. Extracts were neutralized with $2 \mathrm{M} \mathrm{K}_{2} \mathrm{CO}_{3}$, and nucleotides were quantitated by HPLC.

\section{In vivo Slc12a8 knockdown and overexpression.}

To generate shRNA-expressing lentiviral constructs, 56-bp double-stranded oligonucleotides, each of which contains a sense target sequence, a microRNA-based loop sequence (CTTCCTGTCA), an antisense sequence, a termination sequence of four thymidines, and appropriate restriction enzyme sites at both ends, were generated for mouse SIc12a 8 and firefly luciferase $(f L u c)$ and cloned into the U6-PGK-GFP vector provided by the Viral Vectors Core at Washington University School of Medicine. The sense Slc12a8 sequence is '5-5GCCTAGAGTGAACAGAGAAGA-3'. To generate the Slc12a8-expressing lentiviral construct, a 2118-bp fragment of full-length SIc12a8 cDNA was subcloned into the FCIV.FM1 vector provided by the Viral Vectors Core. Lentiviruses were produced by cotransfecting HEK293T cells with the shRNA- or Slc12a8-expressing vectors and three packaging vectors (pMD-Lg, pCMV-G, and RSV-REV) using SuperFect transfection reagent (QIAGEN). Culture supernatant was collected $48 \mathrm{hr}$ after transfection ${ }^{43}$. Knockdown or overexpression efficiencies were tested using primary intestinal cultures ${ }^{44}$. Large-scale lentivirus production was carried out by the Viral Vectors Core at the Hope Center for Neurological Disorders at Washington University. C57BL/6J mice (Jackson Laboratories) orally received $f L u c$ or $S l c 12 a 8$ shRNA lentivirus with a titer of $5 \times 10^{6}$ transduction units or vector-only or Slc12a8-expressing lentivirus with a titer of $3 \times 10^{6}$ transduction units after an overnight fast for two consecutive days.

\section{Generation of antibodies against mouse SIc12a8 and tissue Western blot analysis.}

Two different polyclonal rabbit antisera were produced against a synthetized $\mathrm{N}$-terminal peptide (AQRSPQELFHEAAQQGC) of mouse Slc12a8 (Covance, PA). Mouse tissues were homogenized with RIPA buffer and boiled in 1x Laemmli buffer for 5 min. Western blotting was conducted with a rabbit polyclonal antiserum against the $\mathrm{N}$-terminal portion of mouse Slc12a8 (1:500, Covance, PA) and an anti-Gapdh antibody (1:1000, MAB374, Millipore, MO). Band intensity was quantitated on the Amersham Hyperfilm ${ }^{\text {tm }}$ ECL (GE Healthcare limited) by Photoshop. 


\section{Generation of the whole-body SIc12a8 knockout mice.}

Whole-body SIc12a8 knockout (SIc12a8KO) mice were generated with the CRISPR-CAS9 technology by the Transgenic Vectors Core of Washington University. CRISPR gRNAs were designed to flank exon 4 of the Slc12a8 gene. gRNA sequences were as follows: 5' gRNA; 5' - agtgcatgtatagacgtatg - 3' and 3' gRNA; 5' - cctcacaaatatttacaggc - 3'. gRNAs were obtained as gBlocks (IDT). Cleavage activity was assessed by transfecting N2A cells with gBlock and Cas9 plasmid (addgene \# 42230) using XtremegeneHP (Roche). Cleavage activity was determined by T7E1 assay using standard methods. gRNA was in vitro transcribed using the T7 Megashort Script Kit (Ambion). Cas9 RNA was in vitro transcribed using the mMessage mMachine T7 Ultra Kit (Ambion). All RNA was purified using Megaclear Columns (Ambion). RNA was microinjected into C57BL/6J $\times$ CBA hybrid zygotes at a concentration of $50 \mathrm{ng} / \mu \mathrm{l} \mathrm{Cas} 9,25 \mathrm{ng} / \mu \mathrm{l} \mathrm{gRNA}$, and $100 \mathrm{ng} / \mu \mathrm{ls} \mathrm{sDN}$ in the Washington University Mouse Genetic Core Facility. Whole-body knockout alleles were detected by PCR across the cleavage site and confirmed by sequencing. One heterozygous founder was established, and the mice were backcrossed to wild-type C57BL/6J mice (Jackson Laboratories) for 5 generations before analysis. Slc12a8-deficient heterozygous mice were crossed to generate homozygous Slc12a8KO mice. Wild-type littermates were used as controls.

\section{Production of O18-D-NR and O18-D-NMN.}

${ }^{18} \mathrm{O}$ nicotinamide was prepared from the hydrolysis of cyanopyridine in ${ }^{18} \mathrm{O}$ water ${ }^{45}$. $1,2-{ }^{2} \mathrm{H}, 3,5$-tetraacetate was synthesized from $\mathrm{D}-\left[2-{ }^{2} \mathrm{H}\right]$-ribose (purchased from Omicron Biochemicals) ${ }^{46} \cdot{ }^{18} \mathrm{O}-{ }^{2} \mathrm{H}$ labelled nicotinamide riboside (O18-D-NR) was synthesized from ${ }^{18} \mathrm{O}$-nicotinamide and D-ribofuranose $1,2-{ }^{2} \mathrm{H}, 3,5$-tetraacetate ${ }^{47} \cdot{ }^{18} \mathrm{O}-{ }^{2} \mathrm{H}$ nicotinamide mononucleotide (O18-D-NMN) was synthesized from ${ }^{18} \mathrm{O}-{ }^{2} \mathrm{H}$ nicotinamide riboside, as described previously ${ }^{15}$.

\section{Isotopic tracing experiment.}

$3 \times 10^{5}$ primary hepatocytes isolated from 5 month-old male Slc12a8KO mice and their wildtype littermates were incubated in 6-well plates with $100 \mu \mathrm{M}$ O18-D-NMN or O18-D-NR in DMEM with $1 \%$ FBS for 5 min at $37^{\circ} \mathrm{C} .3 \times 10^{5}$ control and Slc12a8-OE NIH3T3 cells were incubated in 6-well plates with $25 \mu \mathrm{M}$ O18-D-NMN or O18-D-NR in DMEM with $1 \%$ FBS for $5 \mathrm{~min}$ at $37^{\circ} \mathrm{C}$. After incubation, cells were washed twice with cold PBS and lysed in a cold 1:1 mixture of reagent-grade methanol and water. Seven-to-eight month-old Slc12a8KO mice and their wild-type littermates were orally administered with O18-D-NMN at a dose of $500 \mathrm{mg} / \mathrm{kg}$ or PBS after an overnight fast. The jejunum and ileum were collected at $10 \mathrm{~min}$ after oral gavage. A 1:1 mixture of reagent-grade methanol and water $\left(4^{\circ} \mathrm{C}\right)$ were added to the frozen tissue $(60 \mu \mathrm{L} / \mathrm{mg}$ tissue). After sonication, extracts were centrifuged at $12,000 \mathrm{~g}$ for $15 \mathrm{~min}$ at $4^{\circ} \mathrm{C}$. Chloroform was added to the extracts at a ratio of $1: 1(\mathrm{v} / \mathrm{v})$, thoroughly shaken for $30 \mathrm{~s}$, and centrifuged at $12,000 \mathrm{~g}$ for $10 \mathrm{~min}$ at $4^{\circ} \mathrm{C}$. The upper phase (methanol and water) was separated from the lower (organic) phase and lyophilized by speed vacuum at room temperature, reconstituted with $5 \mathrm{mM}$ ammonium formate and centrifuged at $12,000 \mathrm{~g}$ for $10 \mathrm{~min}$. Serial dilutions of NMN, O18-D-NMN, and O18-D-NR at concentrations ranging $128-1000 \mathrm{nmol} / \mathrm{L}$ in $5 \mathrm{mM}$ ammonium formate were used for 
calibration. Liquid chromatography was performed by HPLC (1290; Agilent) with Atlantis T3 (LC $2.1 \times 150 \mathrm{~mm}, 3 \mathrm{~mm}$; Waters) at a flow rate of $0.15 \mathrm{ml} / \mathrm{min}$ with $5 \mathrm{mM}$ ammonium formate for mobile phase A and $100 \%$ methanol for mobile phase B. Metabolites were eluted with gradients of 0-10 min, 0-70\% B; 10-15 min, 70\% B; 16-20 min, 0\% B. The metabolites were analyzed with a Triple Quadrupole mass spectrometer (6470; Agilent) under positive ESI multiple reaction monitoring (MRM) using parameters for NMN (335>123), O18-D-NMN (338>125), and O18-D-NR (258>125). Fragmentation, collision, and post acceleration voltages were $135 \mathrm{~V}, 8 \mathrm{~V}, 7 \mathrm{~V}$ for NMN and $130 \mathrm{~V}, 20 \mathrm{~V}, 1 \mathrm{~V}$ for NR. Peaks of NMN, O18-D-NMN, and O18-D-NR were identified using the MassHunter quantitative analysis tool (Agilent). The areas under the peaks of O18-D-NMN and O18-DNR were calculated by subtracting the background values of PBS controls.

\section{Animal experimentation.}

All mice were group-housed in a barrier facility with $12 \mathrm{hr}$ light/12hr dark cycles. Mice were maintained ad libitum on a standard chow diet (LabDiet 5053; LabDiet, St. Louis, MO). For plasma NMN kinetics and tissue NAD ${ }^{+}$detection, 3-4 month-old male C57BL/6J mice (Jackson Laboratories) that received an oral gavage of $f$ Luc or Slc12a8 shRNA lentivirus, and 3 and 26 month-old female C57BL/6J mice (Charles River) were fasted overnight. Blood was collected from their tail veins at 0,5 and $60 \mathrm{~min}$ after an oral gavage of NMN $(500 \mathrm{mg} / \mathrm{kg}$ ) or PBS. Immediately after blood collection, plasma was separated and quickly extracted with perchloric acid. At this step, it is critical to avoid freezing plasma samples. To show the importance of this procedural precaution, we gave an oral gavage of doubly labeled isotopic NMN (O18-D-NMN) to seventh-month old male C57BL/6J mice (Jackson Laboratories) and measured O18-D-NMN (M+3), O18-NMN (M+2), and NMN (M+0) at 5 min by extracting plasma samples freshly (without freezing/thawing). In this experimental condition, we were able to detect O18-D-NMN $(M+3)$ reliably in plasma samples at $5 \mathrm{~min}$ after oral gavage (Supplementary Fig. 4f), different from the recently reported results ${ }^{23}$. Indeed, extraction of NR from human plasma samples also require a similar caution 22 . Tissue samples were also collected at 60 min time points after an oral gavage of NMN (500 $\mathrm{mg} / \mathrm{kg}$ ) or PBS. NAD ${ }^{+}$levels and Slc12a8 mRNA expression were determined in tissues from 2- or 24-month-old female C57BL/6J mice (Jackson Laboratories) or those in which Slc12a8 was knocked down specifically in the small intestine, as described above. Luminal NMN content was compared by mass spectrometry in the jejunum and ileum between 3 and 26 month-old female C57BL/6J mice (Charles River) fasted overnight. All animal studies were approved by the Washington University Institutional Animal Care and Use Committee and were in accordance with NIH guidelines.

\section{Immunostaining of Slc12a8 in the small intestine.}

Jejuna and Ilea were prepared by the Swiss-roll method and fixed overnight in $10 \%$ buffered formalin (Sigma-Aldrich). Fixed tissues were dehydrated, embedded in paraffin, and sectioned at 5- $\mu \mathrm{m}$ thickness by the Elvie L. Taylor Histology Core Facility of Washington university. Antigen retrieval was performed in sodium citrate buffer $(10 \mathrm{mM}$ Tris-sodium citrate dihydrate [pH 6.0], 0.05\% Tween 20) followed by blocking of endogenous peroxidase activity in $3 \%$ hydrogen peroxide in TBS-T (0.1M Tris- $\mathrm{HCl}$ [pH 7.5], $0.15 \mathrm{M} \mathrm{NaCl}, 0.05 \%$ Tween 20). After blocking with $10 \%$ normal goat serum in TBS-T, samples were incubated 
overnight at $4^{\circ} \mathrm{C}$ with a rabbit anti-mouse-Slc12a 8 antibody (1:100, ARP44039, Aviva, CA) and subsequently with HRP-conjugated goat anti-rabbit $\operatorname{IgG}(1: 1,000)$ antibody using the FITC TSA kit (Parkin Elmer) according to the manufacturer's protocol. Nuclei were stained with DAPI (Sigma \#D9542). Digital images were collected with a fluorescence microscope (ApoTome 2, Zeiss).

\section{Statistical Analyses.}

Differences between two groups were assessed using the unpaired, two-tailed Student's $t$ test. Comparisons among several groups were performed using one-way ANOVA with various post hoc tests indicated in figure legends. $\mathrm{Z}$ ratios and two-sided $p$ values for $S I c 12 a 8$ in Figure 1a were calculated as previously described ${ }^{48}$. For comparisons, $p$ values $<0.05$ were considered statistically significant. All experiments were performed independently at least twice. GraphPad Prism (Version 7) was used to conduct statistical analyses.

\section{Supplementary Material}

Refer to Web version on PubMed Central for supplementary material.

\section{Acknowledgements}

We thank Claudia Cantoni for flow cytometric analysis, Lucrezia Guida for her support in proteoliposome preparation, and Renate Lewis for the production of Slc12a8KO mice. We also thank Mr. Edward Schulak for his generous support to A.G., members of the Imai lab for critical comments and suggestions on this study, and staff members in the core facilities provided by Diabetes Research Center (P30 DK020579), Nutrition Obesity Research Center (P30 DK56341), and the Hope Center for Neurological Disorders at Washington University. This work was also performed in a facility supported by NCRR grant C06 RR015502. A.G. was supported as the Tanaka Scholar by Mr. Tsunemaru Tanaka and Ms. Megumi Tanaka. M.E.M was supported by U.K. Research Councils and Biotechnology and Biological Science Research Council (BBSRC; BB/N001842/1). This work was mainly supported by grants from the National Institute on Aging (AG024150, AG037457, AG047902) to S.I.

\section{References}

1. Canto C, Menzies KJ \& Auwerx J NAD(+) Metabolism and the Control of Energy Homeostasis: A Balancing Act between Mitochondria and the Nucleus. Cell Metab. 22, 31-53, doi:10.1016/j.cmet. 2015.05.023 (2015). [PubMed: 26118927]

2. Rajman L, Chwalek K \& Sinclair DA Therapeutic Potential of NAD-Boosting Molecules: The In Vivo Evidence. Cell Metab 27, 529-547, doi:10.1016/j.cmet.2018.02.011 (2018). [PubMed: 29514064]

3. Verdin E NAD(+) in aging, metabolism, and neurodegeneration. Science 350, 1208-1213, doi: 10.1126/science.aac4854 (2015). [PubMed: 26785480]

4. Yoshino J, Baur JA \& Imai SI NAD(+) Intermediates: The Biology and Therapeutic Potential of NMN and NR. Cell Metab 27, 513-528, doi:10.1016/j.cmet.2017.11.002 (2018). [PubMed: 29249689]

5. Yoshino J, Mills KF, Yoon MJ \& Imai S Nicotinamide mononucleotide, a key NAD(+) intermediate, treats the pathophysiology of diet- and age-induced diabetes in mice. Cell Metab. 14, 528-536, doi:S1550-4131(11)00346-9[pii]10.1016/j.cmet.2011.08.014 (2011). [PubMed: 21982712]

6. Mouchiroud L et al. The NAD(+)/Sirtuin Pathway Modulates Longevity through Activation of Mitochondrial UPR and FOXO Signaling. Cell 154, 430-441, doi:S0092-8674(13)00755-1[pii]10.1016/j.cell.2013.06.016 (2013). [PubMed: 23870130] 
7. Camacho-Pereira J et al. CD38 Dictates Age-Related NAD Decline and Mitochondrial Dysfunction through an SIRT3-Dependent Mechanism. Cell Metab. 23, 1127-1139, doi:10.1016/j.cmet. 2016.05.006 (2016). [PubMed: 27304511]

8. Garten A et al. Physiological and pathophysiological roles of NAMPT and NAD metabolism. Nat. Rev. Endocrinol. 11, 535-546, doi:10.1038/nrendo.2015.117 (2015). [PubMed: 26215259]

9. Imai S Nicotinamide phosphoribosyltransferase (Nampt): a link between NAD biology, metabolism, and diseases. Curr. Pharm. Des. 15, 20-28 (2009). [PubMed: 19149599]

10. Belenky $P$ et al. Nicotinamide riboside promotes Sir2 silencing and extends lifespan via Nrk and Urh1/Pnp1/Meu1 pathways to NAD ${ }^{+}$. Cell 129, 473-484 (2007). [PubMed: 17482543]

11. Caton PW, Kieswich J, Yaqoob MM, Holness MJ \& Sugden MC Nicotinamide mononucleotide protects against pro-inflammatory cytokine-mediated impairment of mouse islet function. Diabetologia 54, 3083-3092, doi:10.1007/s00125-011-2288-0 (2011). [PubMed: 21901281]

12. de Picciotto NE et al. Nicotinamide mononucleotide supplementation reverses vascular dysfunction and oxidative stress with aging in mice. Aging Cell 15, 522-530, doi:10.1111/acel.12461 (2016). [PubMed: 26970090]

13. Gomes AP et al. Declining NAD(+) Induces a Pseudohypoxic State Disrupting NuclearMitochondrial Communication during Aging. Cell 155, 1624-1638, doi:S0092-8674(13)01521-3[pii]10.1016/j.cell.2013.11.037 (2013). [PubMed: 24360282]

14. Long AN et al. Effect of nicotinamide mononucleotide on brain mitochondrial respiratory deficits in an Alzheimer's disease-relevant murine model. BMC Neurol 15, 19, doi:10.1186/ s12883-015-0272-x (2015). [PubMed: 25884176]

15. Mills KF et al. Long-Term Administration of Nicotinamide Mononucleotide Mitigates AgeAssociated Physiological Decline in Mice. Cell Metab. 24, 795-806, doi:10.1016/j.cmet. 2016.09.013 (2016). [PubMed: 28068222]

16. Stein LR \& Imai S Specific ablation of Nampt in adult neural stem cells recapitulates their functional defects during aging. EMBO J. 33, 1321-1340, doi:10.1002/embj.201386917 (2014). [PubMed: 24811750]

17. Wang X, Hu X, Yang Y, Takata T \& Sakurai T Nicotinamide mononucleotide protects against betaamyloid oligomer-induced cognitive impairment and neuronal death. Brain Res 1643, 1-9, doi: 10.1016/j.brainres.2016.04.060 (2016). [PubMed: 27130898]

18. Yamamoto $\mathrm{T}$ et al. Nicotinamide mononucleotide, an intermediate of NAD+ synthesis, protects the heart from ischemia and reperfusion. PLoS One 9, e98972, doi:10.1371/journal.pone.0098972 (2014). [PubMed: 24905194]

19. Ramsey KM, Mills KF, Satoh A \& Imai S Age-associated loss of Sirt1-mediated enhancement of glucose-stimulated insulin secretion in $\beta$ cell-specific Sirt1-overexpressing (BESTO) mice. Aging Cell 7, 78-88 (2008). [PubMed: 18005249]

20. Revollo JR et al. Nampt/PBEF/visfatin regulates insulin secretion in $\beta$ cells as a systemic NAD biosynthetic enzyme. Cell Metab. 6, 363-375 (2007). [PubMed: 17983582]

21. Ratajczak J et al. NRK1 controls nicotinamide mononucleotide and nicotinamide riboside metabolism in mammalian cells. Nat. Commun. 7, 13103, doi:10.1038/ncomms13103 (2016). [PubMed: 27725675]

22. Airhart SE et al. An open-label, non-randomized study of the pharmacokinetics of the nutritional supplement nicotinamide riboside (NR) and its effects on blood NAD+ levels in healthy volunteers. PLoS One 12, e0186459, doi:10.1371/journal.pone.0186459 (2017). [PubMed: 29211728]

23. Liu L et al. Quantitative Analysis of NAD Synthesis-Breakdown Fluxes. Cell Metab. 27, 10671080 e1065, doi:10.1016/j.cmet.2018.03.018 (2018). [PubMed: 29685734]

24. Hebert SC, Mount DB \& Gamba G Molecular physiology of cation-coupled Cl- cotransport: the SLC12 family. Pflugers Arch. 447, 580-593, doi:10.1007/s00424-003-1066-3 (2004). [PubMed: 12739168]

25. Ramsey KM et al. Circadian clock feedback cycle through NAMPT-mediated NAD ${ }^{+}$biosynthesis. Science 324, 651-654, doi:1171641[pii]10.1126/science.1171641 (2009). [PubMed: 19299583] 
26. Yamada K, Hara N, Shibata T, Osago H \& Tsuchiya M The simultaneous measurement of nicotinamide adenine dinucleotide and related compounds by liquid chromatography/electrospray ionization tandem mass spectrometry. Anal. Biochem. 352, 282-285 (2006). [PubMed: 16574057]

27. Shi $\mathrm{W}$ et al. Effects of a wide range of dietary nicotinamide riboside (NR) concentrations on metabolic flexibility and white adipose tissue (WAT) of mice fed a mildly obesogenic diet. Mol Nutr Food Res 61, doi:10.1002/mnfr.201600878 (2017).

28. Sociali $\mathrm{G}$ et al. Antitumor effect of combined NAMPT and CD73 inhibition in an ovarian cancer model. Oncotarget 7, 2968-2984, doi:10.18632/oncotarget.6502 (2016). [PubMed: 26658104]

29. Alessi DR et al. The WNK-SPAK/OSR1 pathway: master regulator of cation-chloride cotransporters. Sci Signal 7, re3, doi:10.1126/scisignal.2005365 (2014). [PubMed: 25028718]

30. Belenky P, Bogan KL \& Brenner C NAD ${ }^{+}$metabolism in health and disease. Trends Biochem. Sci. 32, 12-19 (2007). [PubMed: 17161604]

31. Bieganowski P \& Brenner C Discoveries of nicotinamide riboside as a nutrient and conserved NRK genes establish a Preiss-Handler independent route to NAD+ in fungi and humans. Cell 117, 495-502 (2004). [PubMed: 15137942]

32. Ummarino $\mathrm{S}$ et al. Simultaneous quantitation of nicotinamide riboside, nicotinamide mononucleotide and nicotinamide adenine dinucleotide in milk by a novel enzyme-coupled assay. Food Chem. 221, 161-168, doi:10.1016/j.foodchem.2016.10.032 (2017). [PubMed: 27979136]

33. Hewett $D$ et al. Identification of a psoriasis susceptibility candidate gene by linkage disequilibrium mapping with a localized single nucleotide polymorphism map. Genomics 79, 305-314, doi: 10.1006/geno.2002.6720 (2002). [PubMed: 11863360]

34. Feigin ME et al. Recurrent noncoding regulatory mutations in pancreatic ductal adenocarcinoma. Nat Genet. 49, 825-833, doi:10.1038/ng.3861 (2017). [PubMed: 28481342]

35. Kim JE et al. Associations between genetic polymorphisms of membrane transporter genes and prognosis after chemotherapy: meta-analysis and finding from Seoul Breast Cancer Study (SEBCS). Pharmacogenomics J, doi:10.1038/s41397-018-0016-6 (2018).

36. Wang HH, Patel SB, Carey MC \& Wang DQ Quantifying anomalous intestinal sterol uptake, lymphatic transport, and biliary secretion in Abcg8(-/-) mice. Hepatology 45, 998-1006, doi: 10.1002/hep.21579 (2007). [PubMed: 17393508]

37. Yoshino J \& Imai S Accurate Measurement of Nicotinamide Adenine Dinucleotide (NAD(+)) with High-Performance Liquid Chromatography. Methods Mol. Biol. 1077, 203-215, doi: 10.1007/978-1-62703-637-5_14 (2013). [PubMed: 24014409]

38. Grimm AA, Brace CS, Wang T, Stormo GD \& Imai S A nutrient-sensitive interaction between Sirt1 and HNF-1alpha regulates Crp expression. Aging Cell 10, 305-317, doi:10.1111/j. 1474-9726.2010.00667.x (2011). [PubMed: 21176092]

39. Revollo JR, Grimm AA \& Imai S The NAD biosynthesis pathway mediated by nicotinamide phosphoribosyltransferase regulates Sir2 activity in mammalian cells. J. Biol. Chem. 279, 5075450763 (2004). [PubMed: 15381699]

40. Bruzzone $\mathrm{S}$ et al. The plant hormone abscisic acid increases in human plasma after hyperglycemia and stimulates glucose consumption by adipocytes and myoblasts. FASEB J. 26, 1251-1260, doi: 10.1096/fj.11-190140 (2012). [PubMed: 22075645]

41. Bruzzone S, Guida L, Zocchi E, Franco L \& De Flora A Connexin 43 hemi channels mediate Ca2+-regulated transmembrane NAD+ fluxes in intact cells. FASEB J. 15, 10-12, doi:10.1096/fj. 00-0566fje (2001). [PubMed: 11099492]

42. Franco L et al. The transmembrane glycoprotein CD38 is a catalytically active transporter responsible for generation and influx of the second messenger cyclic ADP-ribose across membranes. FASEB J. 12, 1507-1520 (1998). [PubMed: 9806760]

43. Satoh A et al. Sirt1 Extends Life Span and Delays Aging in Mice through the Regulation of Nk2 Homeobox 1 in the DMH and LH. Cell Metab. 18, 416-430, doi:S1550-4131(13)00333-1[pii]10.1016/j.cmet.2013.07.013 (2013). [PubMed: 24011076]

44. Sato T \& Clevers H Primary mouse small intestinal epithelial cell cultures. Methods Mol. Biol. 945, 319-328, doi:10.1007/978-1-62703-125-7_19 (2013). [PubMed: 23097115]

45. Kolodziejska-Huben M, Kaminski Z \& Paneth P Preparation of 18O-labelled nicotinamide. J. Label. Compd. Radiopharm. 45, 1005-1010, doi:10.1002/jlcr.617 (2002). 
46. Chatterjee A, Hazra AB, Abdelwahed S, Hilmey DG \& Begley TPA "radical dance" in thiamin biosynthesis: mechanistic analysis of the bacterial hydroxymethylpyrimidine phosphate synthase. Angew. Chem. Int. Ed. Engl. 49, 8653-8656, doi:10.1002/anie.201003419 (2010). [PubMed: 20886485]

47. Fouquerel E et al. ARTD1/PARP1 negatively regulates glycolysis by inhibiting hexokinase 1 independent of NAD+ depletion. Cell Rep. 8, 1819-1831, doi:10.1016/j.celrep.2014.08.036 (2014). [PubMed: 25220464]

48. Cheadle C, Vawter MP, Freed WJ \& Becker KG Analysis of microarray data using Z score transformation. J. Mol. Diagn. 5, 73-81, doi:10.1016/S1525-1578(10)60455-2 (2003). [PubMed: 12707371] 
a

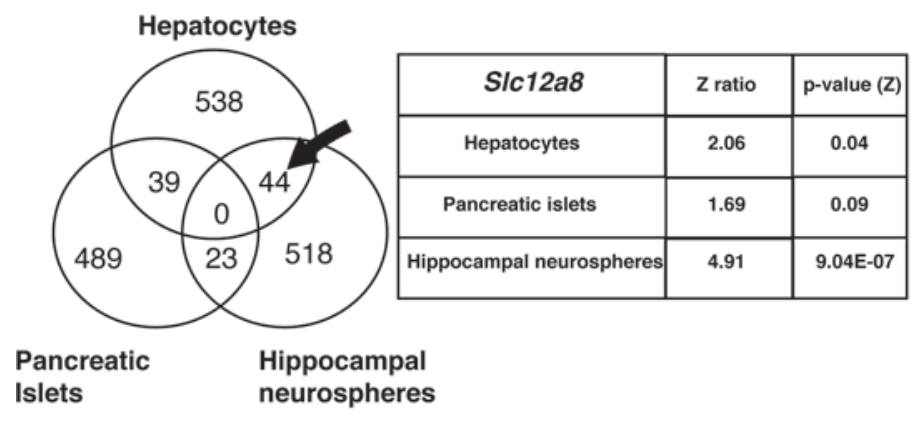

b

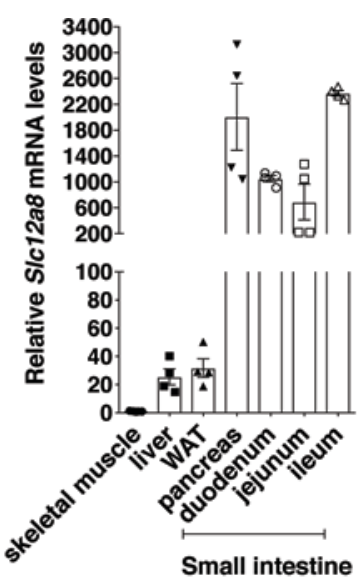

C

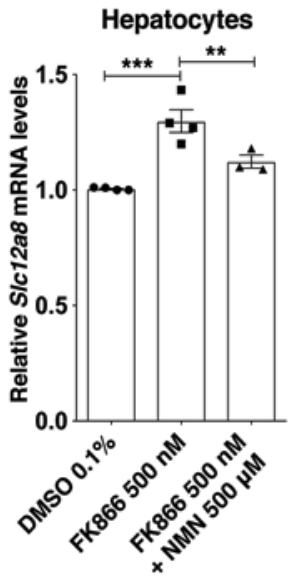

d

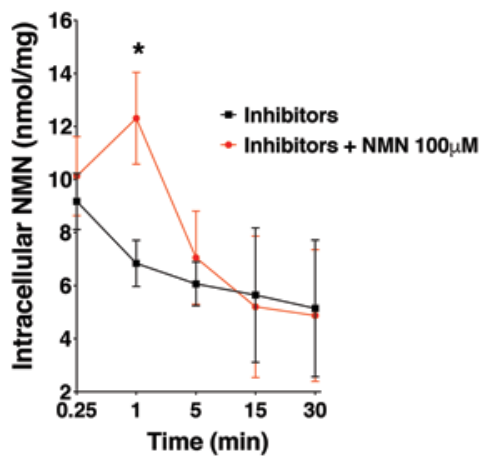

NIH3T3

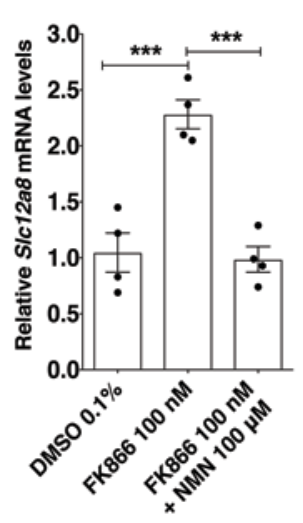

e
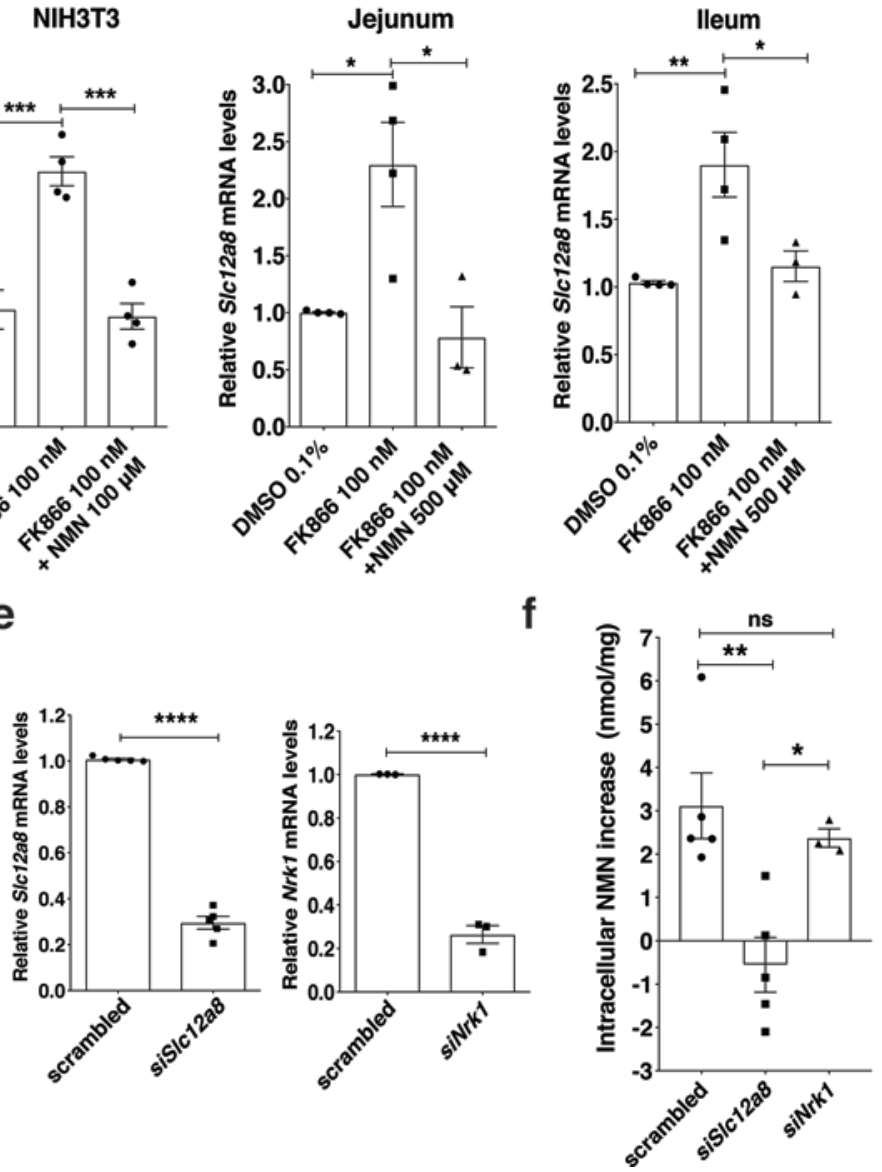

Figure 1.

Identification and characterization of the Slc12a8 gene. a, Venn diagram of genes commonly upregulated in primary hepatocytes, pancreatic islets, and hippocampal neurospheres treated with FK866. Slc12a8 was identified in the section indicated by an arrow. Z ratios and twosided $p$ values for SIc12a 8 in each cell type were calculated as described in the Methods section ( $\mathrm{n}=4$ biologically independent samples). b, Relative SIc12a8 mRNA levels in different tissues from B6 male mice at 3 months of age ( $n=4$ mice). WAT, white adipose tissue. c, Relative Slc12a 8 mRNA levels in primary mouse hepatocytes ( $\mathrm{n}=4$ mice, ** 
$\mathrm{p}=0.0191, * * * \mathrm{p}=0.0006)$, NIH3T3 fibroblasts ( $\mathrm{n}=4$ biologically independent samples, DMSO vs. FK866 ***p=0.0004, FK866 vs. FK866 plus NMN ***p=0.0003), and ex vivo explants of the jejunum and ileum ( $\mathrm{n}=4$ mice for DMSO and FK866 alone; $\mathrm{n}=3$ mice for FK866 plus NMN; Jejunum, DMSO vs. FK866 *p=0.0168, FK866 vs. FK866+NMN * $\mathrm{p}=0.0111$; Ileum, DMSO vs. FK866 ** $\mathrm{p}=0.009$, FK866 vs. FK866+NMN *p=0.0313) treated with $0.1 \%$ DMSO, FK866 alone or FK866 plus NMN (24h for cells, and $4 \mathrm{~h}$ for explants; analyzed using ANOVA with Tukey's test). d, Time course of NMN uptake in mouse primary hepatocytes. Hepatocytes were pretreated with $500 \mathrm{nM} \mathrm{FK} 866$ for $24 \mathrm{~h}$ and then incubated with a cocktail of $20 \mu \mathrm{M}$ dipyridamole, $500 \mu \mathrm{M}$ AOPCP, and $500 \mathrm{nM}$ FK866, with or without $100 \mu \mathrm{M}$ NMN. NMN was measured by HPLC ( $\mathrm{n}=4$ mice, except for 3 data sets for 15 and 30 time points for inhibitors only; analyzed using ANOVA with Sidak's test, $* \mathrm{p}=0.0262$ ). e, Knockdown efficiencies of Slc12a 8 and $N_{r k} 1$ mRNA in mouse primary hepatocytes ( $\mathrm{n}=5$ mice for $S 1 \mathrm{c} 12 \mathrm{a} 8$ silencing and $\mathrm{n}=3$ for $N r k 1$ silencing; analyzed by unpaired two-sided $t$-test, siSlc12a $8 * * * \mathrm{p}<0.0001$, si $N r k 1 * * * * \mathrm{p}<0.0001)$. f, Increases in intracellular NMN content measured by HPLC in primary hepatocytes treated with scrambled, Slc12a8, and Nrk1 siRNA at 1 min after addition of $100 \mu \mathrm{M}$ NMN. Culture conditions were the same as described in $\mathbf{d}$ ( $\mathrm{n}=5$ mice for 51 1 $12 \mathrm{a} 8$ silencing and $\mathrm{n}=3$ for Nrk1 silencing; analyzed by ANOVA with Tukey's test, $\left.{ }^{* *} \mathrm{p}=0.0052,{ }^{*} \mathrm{p}=0.0413\right)$. All values are presented as mean \pm SEM. 

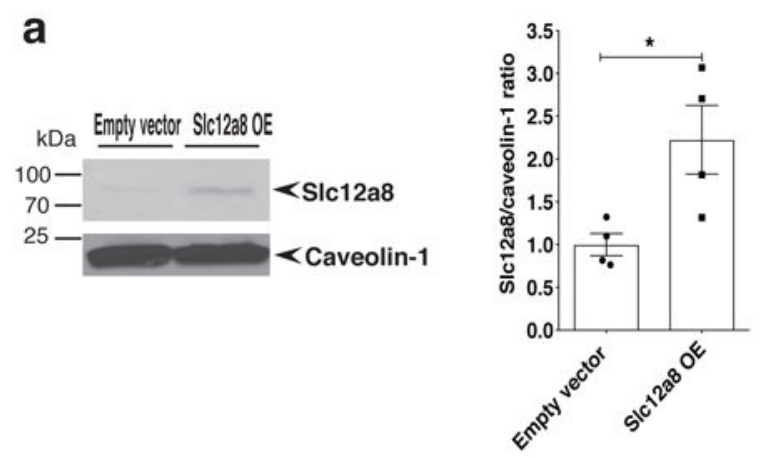

b

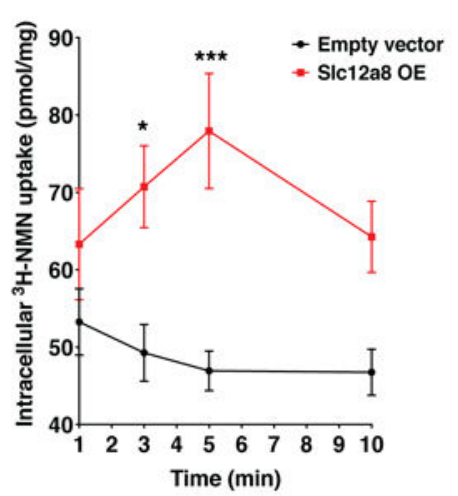

C

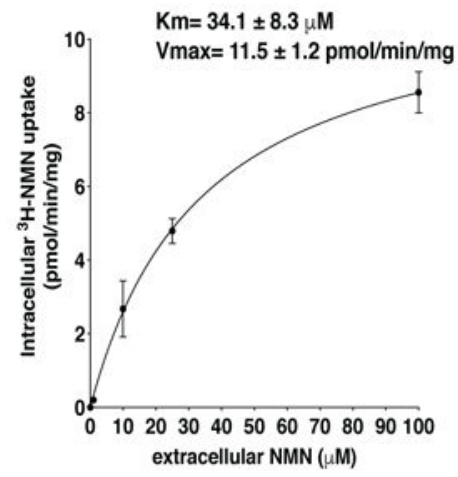

d

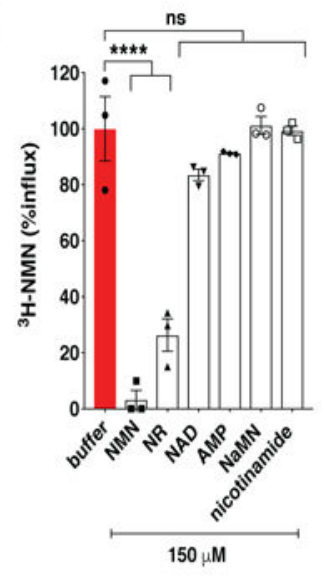

e

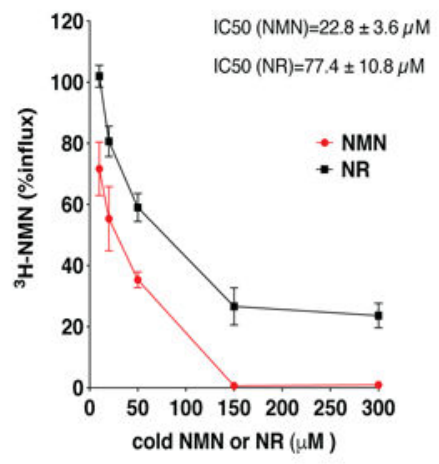

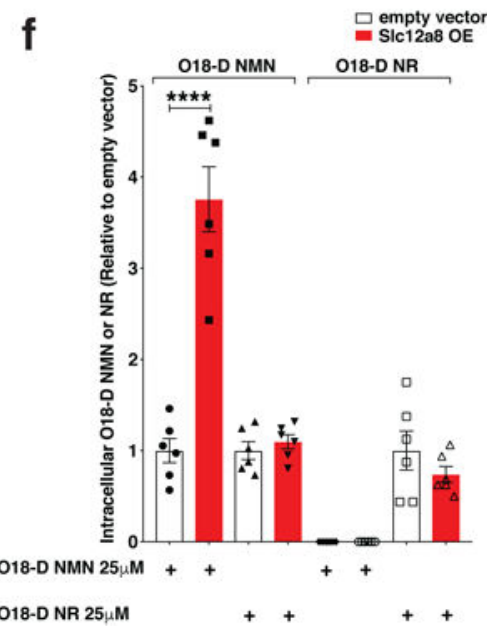
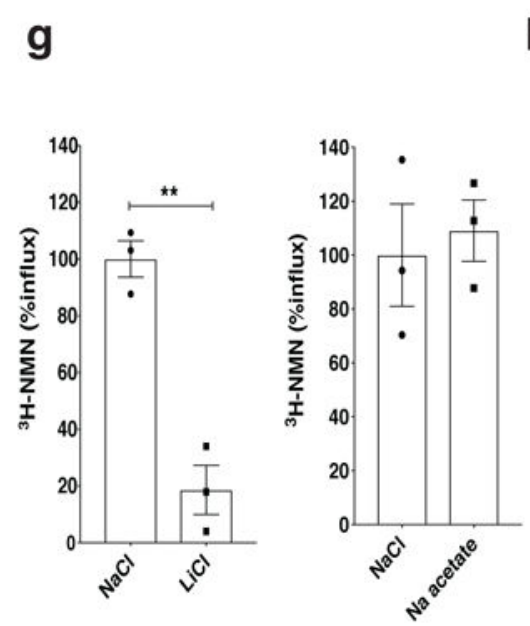

h

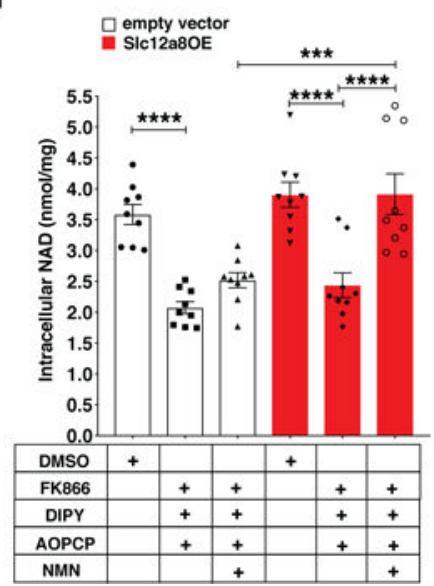

Figure 2.

The kinetic features of the Slc12a8 NMN transporter and its specificity, sodium dependency, and effects on $\mathrm{NAD}^{+}$biosynthesis. a, Slc12a8 protein expression in plasma membrane fractions from control and Slc12a8-OE NIH3T3 cells (left panel). Slc12a8 protein levels normalized to caveolin-1 protein levels are shown for each cell line (right panel; $n=4$ independent experiments; analyzed by unpaired two-sided $t$-test, $\left.{ }^{*} \mathrm{p}=0.0271\right)$. bo Uptake of ${ }^{3} \mathrm{H}$-labeled NMN $\left({ }^{3} \mathrm{H}-\mathrm{NMN} ; 25 \mu \mathrm{M}, 37^{\circ} \mathrm{C}\right.$ ) in control and Slc12a8-OE NIH3T3 cells ( $\mathrm{n}=12$ biologically independent samples; analyzed by ANOVA with Sidak's test, * $\mathrm{p}=0.0136$, 
*** $\mathrm{p}=0.0001)$. c, $K_{m}$ and $V_{\max }$ of Slc12a8 for NMN transport. Those values were determined by non-linear regression analysis by subtracting the backgrounds of control cells ( $n=5$ biologically independent samples for 1 and $10 \mu \mathrm{M}$, and $\mathrm{n}=4$ biologically independent samples for 25 and $100 \mu \mathrm{M}$ ). d, Substrate specificity of Slc12a8. Transport of ${ }^{3} \mathrm{H}-\mathrm{NMN}$ (150 $\mathrm{nM}, 25^{\circ} \mathrm{C}$ ) into proteoliposomes derived from Slc12a8-OE cells was measured at $2 \mathrm{~min}$ in the presence of competing cold compounds ( $\mathrm{n}=3$ biologically independent samples, analyzed by ANOVA with Dunnett's test, ${ }^{* * * *} \mathrm{p}=0.0001$; ns, not significant). e, The half maximal inhibitory concentrations $\left(\mathrm{IC}_{50}\right)$ of NMN and NR. Data are shown as percentages of ${ }^{3} \mathrm{H}$ NMN uptake ( $\mathrm{n}=3$ biologically independent samples; $\mathrm{IC}_{50}$ was calculated by non-linear regression analysis). f, Intracellular levels of doubly labeled, isotopic NMN (O18-D-NMN) and NR (O18-D-NR) were measured by mass spectrometry in control and Slc12a8-OE NIH3T3 cells incubated with $25 \mu \mathrm{M}$ O18-D-NMN or O18-D-NR for $5 \mathrm{~min}$ ( $\mathrm{n}=6$ biologically independent samples; analyzed by unpaired two-sided $t$-test, $* * * * p<0.0001)$. Values are expressed relative to O18-D-NMN or O18-D-NR levels detected in control NIH3T3 cells. g, Ion dependency of NMN uptake by Slc12a8. Sodium ion $\left(\mathrm{Na}^{+}\right)$or chloride ion $\left(\mathrm{Cl}^{-}\right)$was replaced with an equimolar concentration of lithium $\left(\mathrm{Li}^{+}\right)$or acetate, respectively $(\mathrm{n}=3$ biologically independent samples, analyzed by unpaired two-sided $t$-test, $* * \mathrm{p}=0.0017) . \mathbf{h}$, Intracellular $\mathrm{NAD}^{+}$content was measured as described in the Methods section ( $\mathrm{n}=9$ biologically independent samples; analyzed by ANOVA with Tukey's test; control, DMSO vs inhibitors $* * * * \mathrm{p}<0.0001$; Slc12a8-OE, DMSO vs. inhibitors $* * * * \mathrm{p}<0.0001$, inhibitors vs. inhibitors $+\mathrm{NMN} * * * * \mathrm{p}<0.0001$; control, inhibitors $+\mathrm{NMN}$ vs. Slc12a8-OE, inhibitors $+\mathrm{NMN}$ $* * * \mathrm{p}=0.0002$ ). All values are presented as mean \pm SEM. 
a

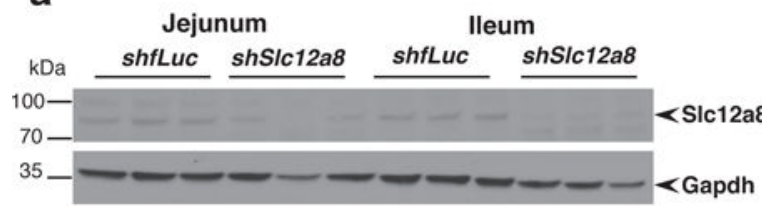

b

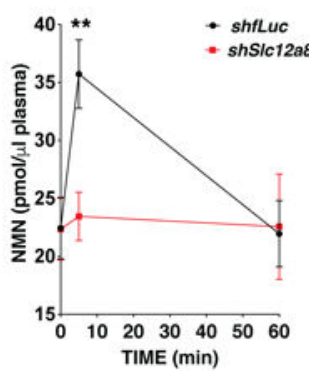

C

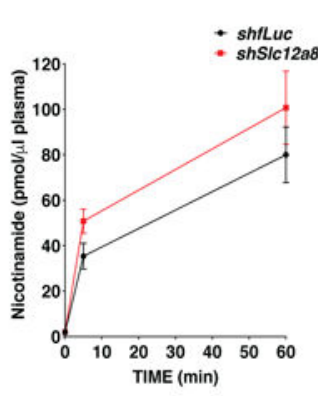

d
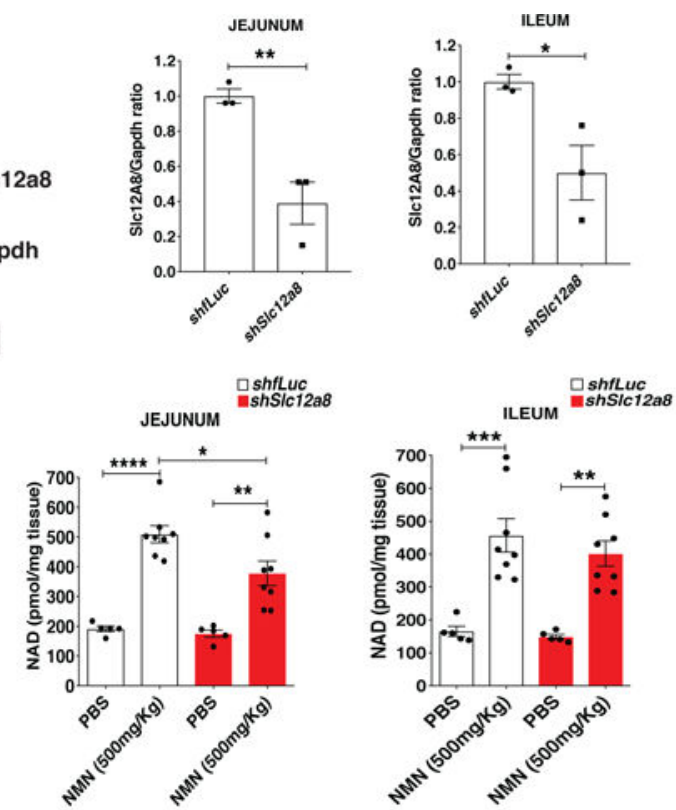

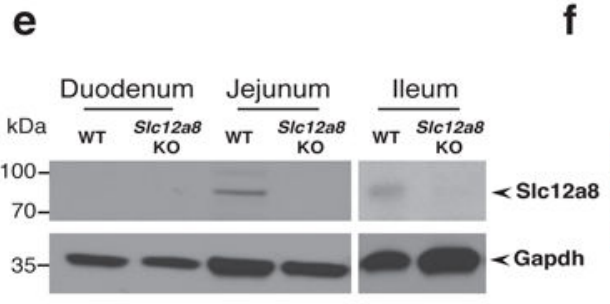

g

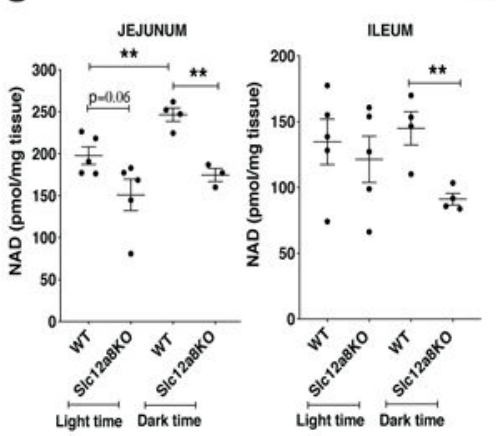

h

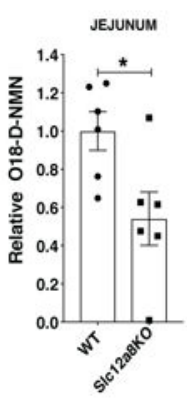

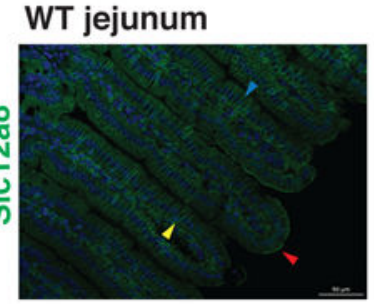

i SIc12a8 KO jejunum

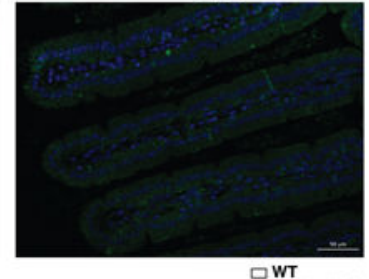

SIc12a8ko
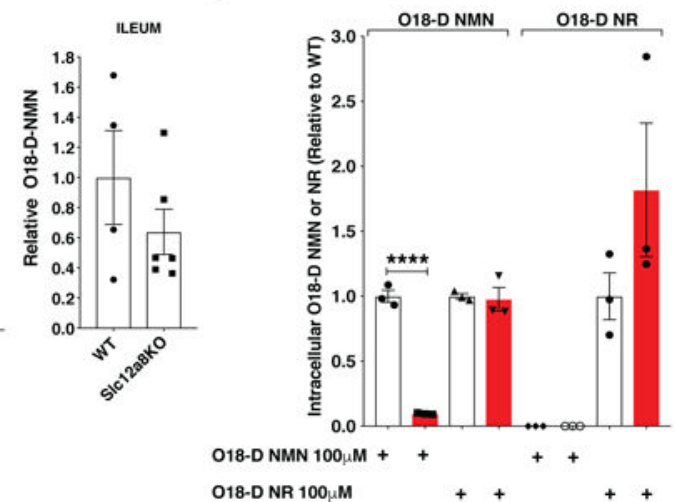

Figure 3.

The in vivo knockdown (KD) of Slc12a8 in the small intestine. a, Slc12a8 protein levels in control and Slc12a8 KD jejunum and ileum samples. A representative Western blot is shown (left panel), and bar graphs show Slc12a8 protein levels normalized to Gapdh protein levels (right panel) ( $\mathrm{n}=3$ mice, repeated twice; B6 males at 3-4 months of age; analyzed by unpaired two-sided $t$-test, $* \mathrm{p}=0.0324, * * \mathrm{p}=0.0085)$. b, Plasma NMN levels after an oral gavage of NMN (500 mg/kg body weight) in control and Slc12a8 KD mice ( $\mathrm{n}=6$ mice; B6 males at 3-4 months of age; analyzed by ANOVA with Sidak's test, **p=0.0080). c, Plasma 
nicotinamide levels in the same mice described in $\mathbf{b}\left(\mathrm{n}=6\right.$ mice). $\mathbf{d}$, Tissue NAD ${ }^{+}$levels in the jejunum and ileum samples collected at $60 \mathrm{~min}$ time point after an oral gavage, as described in $\mathbf{b}$ ( $\mathrm{n}=5$ mice for PBS, and $\mathrm{n}=8$ for NMN; B6 males at 3-4 months of age; analyzed by ANOVA with Tukey's test; Jejunum, shfLuc PBS vs. NMN **** p $<0.0001$, shSlc12a8 PBS vs. NMN **p=0.0029, shfLuc NMN vs. shSlc12a8 NMN *p=0.0029; Ileum, shfLuc PBS vs. NMN ***p=0.0003, shSlc12a8 PBS vs. NMN **p=0.0030 ). e, Slc12a8 and Gapdh proteins in tissue lysates of the duodenum, jejunum, and ileum of Slc12a8KO mice and wild-type littermates (WT) ( $\mathrm{n}=3$ mice). f, Immunostaining of Slc12a8 (green) in the jejunum from 10 month-old Slc12a8KO female mice and WT littermates (n=3 mice). Red, blue, and yellow arrowheads indicate apical, lateral and basal membranes, respectively. Scale bars: $50 \mu \mathrm{m}$. g, Tissue $\mathrm{NAD}^{+}$levels in the jejunum and ileum from Slc12a8KO mice and WT littermates, collected during light time (9-10 am) or during dark time (9-10 pm) ( $n=5$ mice for the light time, and $n=4$ mice for the dark time, except for the 3 data points for the jejunum of Slc12a8KO mice; females at 8-10 months of age; analyzed by unpaired twosided $t$-test; Jejunum, dark time WT vs. Slc12a $8 \mathrm{KO} * * \mathrm{p}=0.0015$, light time WT vs. dark time WT **p=0.0093; Ileum, dark time WT vs. Slc12a8KO **p=0.0069). h, Levels of doubly labeled, isotopic NMN (O18-D-NMN) in the jejunum and ileum by mass spectrometry at $10 \mathrm{~min}$ after orally administering $500 \mathrm{mg} / \mathrm{kg}$ of O18-D-NMN in $51 \mathrm{c} 12 \mathrm{a} 8 \mathrm{KO}$ mice and WT littermates ( $\mathrm{n}=6$ mice, 3 males and 3 females at 7-8 months of age, except for 2 males and 2 females for the wild-type ileum; analyzed by unpaired two-sided $t$-test, * $\mathrm{p}=0.0239$ ). Values are expressed relative to O18-D-NMN levels detected in WT. i, Intracellular levels of O18-D-NMN and O18-D-NR by mass spectrometry in primary hepatocytes isolated from 5 month-old S/c12a8KO male mice and WT littermates and incubated with $100 \mu \mathrm{M}$ O18-D-NMN or O18-D-NR for $5 \mathrm{~min}$ ( $\mathrm{n}=3$ mice; analyzed by unpaired two-sided $t$-test, $* * * * \mathrm{p}<0.0001)$. Values are expressed relative to O18-D-NMN or O18-D-NR levels detected in WT. All values are presented as mean \pm SEM. 
a

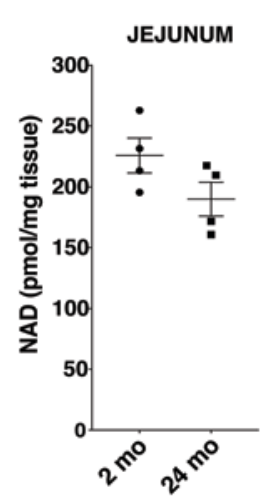

C
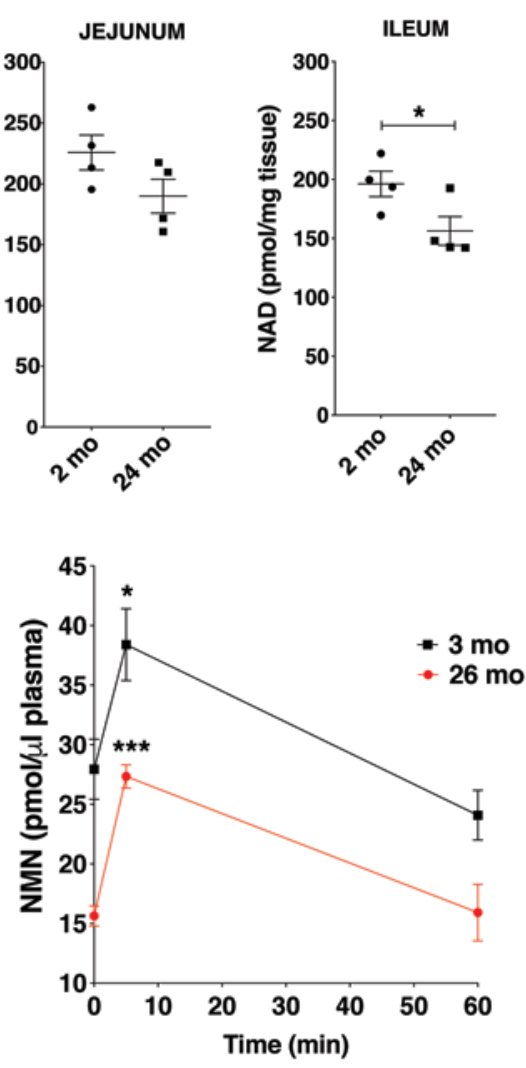

b
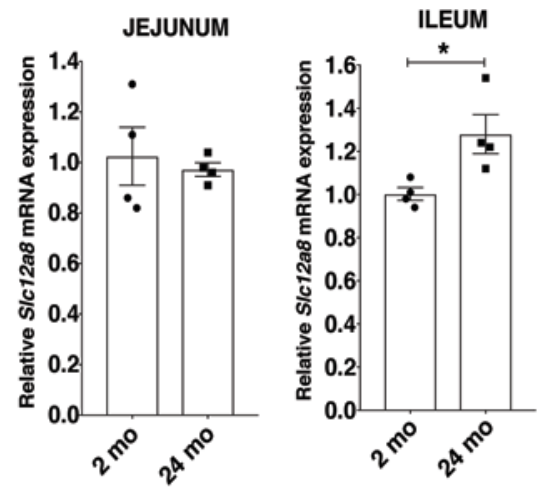

d

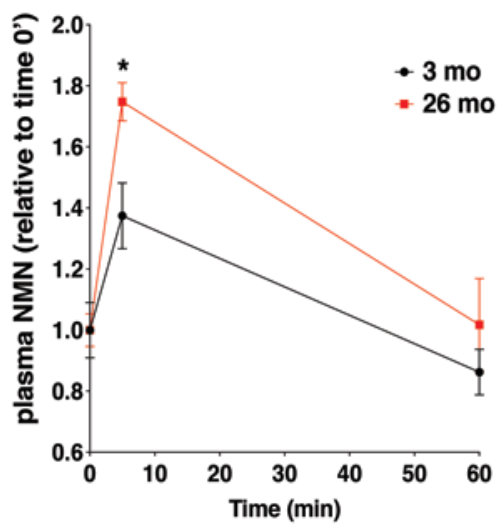

e

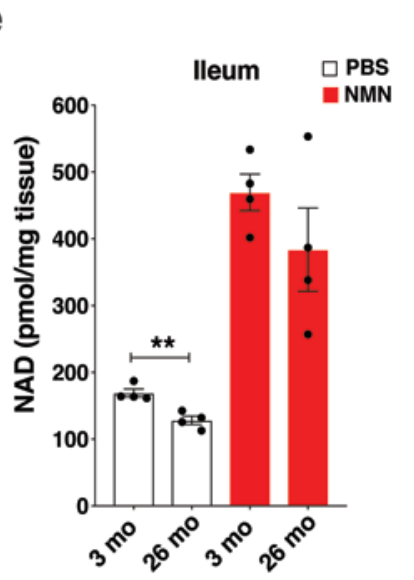

f

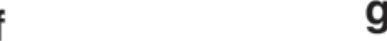

g
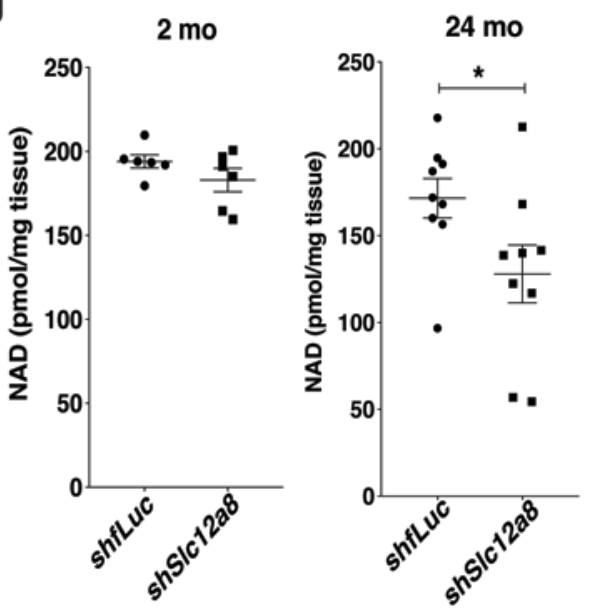

Figure 4.

The age-associated upregulation of S/c12a8 in the ileum and its effect on NMN uptake and ileal $\mathrm{NAD}^{+}$biosynthesis in aged mice. a, b, NAD ${ }^{+}$levels (a) and relative Slc12a8 mRNA levels (b) in the jejuna and ilea from 2 month-old (2 mo) and 24-month-old (24 mo) B6 female mice. Tissue samples were collected during the dark time $(9-10 \mathrm{pm})(\mathrm{n}=4$ mice for each age, analyzed by unpaired two-sided $t$-test; NAD ${ }^{+}$levels, *p=0.0492; Slc12a8 mRNA levels, $\left.{ }^{*} \mathrm{p}=0.0269\right)$. c, $\mathbf{d}$, Plasma NMN levels (c) and their relative changes (d) after an oral gavage of NMN (500 mg/kg body weight) in 3 month-old ( $3 \mathrm{mo}$ ) and 26 month-old (26 mo) 
B6 female mice ( $\mathrm{n}=4$ mice; analyzed by ANOVA with Sidak's test; $\mathbf{c}$, ${ }^{*} \mathrm{p}=0.0328$, $* * * \mathrm{p}=0.0010 ; \mathbf{d}, * \mathrm{p}=0.0257)$. e, f, Ileal $\mathrm{NAD}^{+}$levels $(\mathbf{e})$ and their relative comparisons $(\mathbf{f})$ in the ileal samples collected at $60 \mathrm{~min}$ time point after an oral gavage in 3 month-old ( $3 \mathrm{mo}$ ) and 26 month-old ( 26 mo) B6 female mice ( $\mathrm{n}=4$ mice each for PBS and NMN, analyzed by unpaired two-sided $t$-test; $\left.\mathbf{e},{ }^{* *} \mathrm{p}=0.0033 ; \mathbf{f}, * * \mathrm{p}=0.0066 * * * * \mathrm{p}<0.0001\right)$. g, NAD ${ }^{+}$levels in the ilea of 2 and 24 month-old control and intestinal SIc12a8 KD B6 female mice (n=6 mice each for 2 month-old control and intestinal Slc12a8-KD B6 female mice, and n=9 mice each for 24 month-old control and intestinal Slc12a8 KD B6 female mice; analyzed by unpaired two-sided $t$-test, $\left.{ }^{*} \mathrm{p}=0.0496\right)$. All values are presented as mean $\pm \mathrm{SEM}$. 